\title{
A mid-IR interferometric survey with MIDI/VLTI: resolving the second-generation protoplanetary disks around post-AGB binaries *
}

\author{
M. Hillen ${ }^{1}$, H. Van Winckel ${ }^{1}$, J. Menu ${ }^{1}$, R. Manick ${ }^{1}$, J. Debosscher ${ }^{1}$, M. Min ${ }^{2,3}$, W.-J. de Wit ${ }^{4}$, T. Verhoelst ${ }^{5}$, \\ D. Kamath ${ }^{1}$, and L. B. F. M. Waters ${ }^{2}$
}

1 Instituut voor Sterrenkunde (IvS), KU Leuven, Celestijnenlaan 200D, 3001 Leuven, Belgium e-mail: Michel.Hillen@ster.kuleuven.be

2 Anton Pannekoek Institute for Astronomy, University of Amsterdam, 1090 GE Amsterdam, The Netherlands

3 SRON, Netherlands Institute for Space Research, Sorbonnelaan 2, 3584 CA Utrecht, The Netherlands

4 European Southern Observatory, Alonso de Cordova 3107, Vitacura, Santiago, Chile

5 Belgian Institute for Space Aeronomy, Brussels, Belgium

${ }^{6}$ SRON, Netherlands Institute for Space Research, Sorbonnelaan 2, 3584 CA Utrecht, The Netherlands

Received 21 June 2016 / Accepted 14 September 2016

\begin{abstract}
Aims. We present a mid-IR interferometric survey of the circumstellar environment of a specific class of post-asymptotic giant branch (post-AGB) binaries. For this class the presence of a compact dusty disk has been postulated on the basis of various spatially unresolved measurements. The aim is to determine the angular extent of the $\mathrm{N}$-band emission directly and to resolve the compact circumstellar structures.

Methods. Our interferometric survey was performed with the MIDI instrument on the VLTI. In total 19 different systems were observed using variable baseline configurations. Combining all the visibilities at a single wavelength at $10.7 \mu \mathrm{m}$, we fitted two parametric models to the data: a uniform disk and a ring model mimicking a temperature gradient. We compared our observables of the whole sample, with synthetic data computed from a grid of radiative transfer models of passively irradiated disks in hydrostatic equilibrium. These models are computed with a Monte Carlo code that has been widely applied to describe the structure of protoplanetary disks around young stellar objects (YSO).

Results. The spatially resolved observations show that the majority of our targets cluster closely together in the distance-independent size-colour diagram, and have extremely compact $N$-band emission regions. The typical uniform disk diameter of the $N$-band emission region is $\sim 40$ mas, which corresponds to a typical brightness temperature of 400-600 K. The resolved objects display very similar characteristics in the interferometric observables and in the spectral energy distributions. Therefore, the physical properties of the disks around our targets must be similar. Our results are discussed in the light of recently published sample studies of YSOs to compare quantitatively the secondary discs around post-AGB stars to the ones around YSOs.

Conclusions. Our high-angular-resolution survey further confirms the disk nature of the circumstellar structures present around wide post-AGB binaries. The grid of protoplanetary disk models covers very well the observed objects. Much like for young stars, the spatially resolved $N$-band emission region is determined by the hot inner rim of the disk. Continued comparisons between post-AGB and protoplanetary disks will help to understand grain growth and disk evolution processes, and to constrain planet formation theories. These second-generation disks are an important missing ingredient in binary evolution theory of intermediate-mass stars.
\end{abstract}

Key words. stars: AGB and post-AGB - binaries: spectroscopic - techniques: high angular resolution - techniques: interferometric circumstellar matter

\section{Introduction}

The impact of binarity on the evolution of low- to intermediatemass stars is an important, yet poorly understood, domain of stellar astrophysics. Interactions between the loosely bound envelope of a (super)giant and the gravitational pull of its companion result in a diverse zoo of peculiar objects. Binarity is not only responsible for spectacular phenomena like supernovae type Ia,

\footnotetext{
* Based on observations made with ESO Telescopes at the La Silla Paranal Observatory under programmes ID 073.A-9002, 073.A-9014, 073.D-0610, 075.D-0605, 077.D-0071，078.D-0113，079.D-0013, 080.D-0059, 081.D-0089, 082.D-0066， 083.D-0011， 083.D-0013, 084.D-0009, 093.D-0914, and 094.D-0778. Some observations were obtained in the framework of the Belgian Guaranteed Time allocation on VISA.
}

sub-luminous supernovae, gravitational wave sources and novae explosions, but also for less energetic systems like sub-dwarf B stars, barium stars and cataclysmic variables. Furthermore, binary interaction processes influence the shaping of planetary nebulae (PNe). Therefore, binary interaction physics plays a fundamental role in understanding stellar evolution.

In this work we focus on the circumstellar environment (CE) of a distinct class of evolved objects. All sample objects show a characteristic strong near-IR excess which can be explained as due to thermal emission of warm dust in the close environment around the central binary. The energy source in these systems is an evolved low-mass object in its post-asymptotic giant branch (post-AGB) phase of evolution (van Winckel 2003; de Ruyter et al. 2006), with a typical spectral type between G 
and late A. The characteristic spectral energy distribution (SED) in these objects indicates the presence of a stable, compact, dusty reservoir in the form of a circumbinary disk (e.g. de Ruyter et al. 2006; Deroo et al. 2007; Hillen et al. 2014, 2015). The Keplerian rotation of the circumstellar structure was already resolved in two objects Bujarrabal et al. $(2005,2015)$ and recently much more detailed maps were made with the Atacama Large Millimetre Array (ALMA) by Bujarrabal et al. (2013b). The singledish CO line survey of Bujarrabal et al. (2013a) confirmed, by the detection of very narrow $\mathrm{CO}$ line profiles, that rotation is likely widespread, which is a strong observational indicator of long-term stability. Other indications of longevity are the observational evidence of strong dust grain processing in the form of a high degree of crystallinity (e.g. Gielen et al. 2008, 2011) and the presence of large grains (e.g. de Ruyter et al. 2005).

These specific SEDs can be intimately linked to the binary nature of the central object. The luminous evolved component has a likely unevolved companion with a very minor contribution to the energy budget (e.g. van Winckel et al. 2009; Hillen et al. 2016). Based on the SED, new such objects can be efficiently identified. In recent searches for post-AGB stars in the Large and Small Magellanic Clouds (van Aarle et al. 2011; Kamath et al. 2014, 2015), disk sources represent about half of the population of optically bright post-AGB stars. Disks also appear at lower luminosities, indicating them to be post-Red Giant Branch (postRGB) stars, rather than post-AGB stars (Kamath et al. 2016). Evolved sources with these specific SED characteristics are very common, and the formation, structure, and evolution of these disks represents an important gap in our understanding of binary interaction physics.

The inner rim is an important structural component of a postAGB disk, just like in young stellar objects (YSO). The rim captures the radiation of the central source and gas pressure makes that the scale height becomes large. A significant fraction of the stellar luminosity is re-radiated by the disk in the infrared (e.g. Dullemond \& Monnier 2010). As the disks are compact, interferometric tools are needed to resolve them. This is nicely illustrated by our recent study of one of the evolved binaries, IRAS 08544-4431 (Hillen et al. 2016). Using an interferometric image reconstruction technique, Hillen et al. (2016) resolved the inner circumstellar environment of IRAS 08544-4431. The image, with a spatial resolution of 1.3 mas, shows that the inner rim of the disk is indeed at the sublimation radius. Moreover, a contribution of the companion star is spatially resolved and likely originating from a small gas accretion disk (Hillen et al. 2016).

Here we aim at constraining the disk properties for a significant number of stars. We report on the first $N$-band interferometric survey of these binary post-AGB stars, providing direct insight into the spatial structure of the circumstellar environment. The observations were performed with the MID-infrared Interferometric instrument (MIDI, Leinert et al. 2003) on the Very Large Telescope Interferometer (Haguenauer et al. 2012). MIDI operates in the $\mathrm{N}$-band, which is where the circumstellar environment emits the peak of its energy. Several case studies were presented in previous papers (e.g. Hillen et al. 2014, 2015, and references therein). These results inspired our survey of $19 \mathrm{ob}-$ jects. The intent of this paper is to gain insight into the overall sample characteristics and study the typical physical scales of the disks. The challenge of this work is that many objects are observed, but each with a limited baseline coverage. Recently, similar surveys with MIDI were published about the torii in active galactic nuclei (Burtscher et al. 2013), the structures around massive YSOs (Boley et al. 2013), and the circumstellar disks around Herbig Ae/Be stars (Menu et al. 2015). Our methodology is heavily inspired by the last, which allows us to make a direct comparison between the two samples.

The paper is organized as follows: first, we describe the different programme stars (Sect. 2) and their data (Sect. 3). We report on our construction of detailed SEDs for all objects in Sect. 4 and on the fitting of the interferometric observables in Sect. 5. The results on some individual objects are highlighted in Sect. 6 and we analyse the sample as a whole in Sect. 7. We confront the interferometric observables with the results of a grid of radiative transfer models in Sect. 8 and focus on comparing the circumbinary structures around our evolved binaries with the circumstellar structures in YSOs. Finally, we discuss the implications of our results and conclude the paper in Sect. 9.

\section{Description of the sample}

The sample of Galactic post-AGB stars for which we obtained $N$-band interferometric data is listed in Table 1 . The sample of 19 stars was selected from the catalogue of de Ruyter et al. (2006) of post-AGB objects with a suspected circumstellar disk, with the addition of HD 101584 (Bakker et al. 1996). The primary criteria to select the science targets to be observed with MIDI were the estimated correlated $N$-band flux-level as well as the accessibility from the Paranal observatory. We have used both the Unit Telescopes (UT, $8 \mathrm{~m}$ diameter) and the Auxiliary Telescopes (AT, $1.8 \mathrm{~m}$ diameter), allowing the inclusion of targets with both moderate and high $N$-band fluxes. About half of our sources were observed within the Belgian Guaranteed Time programme (VISA) on the ATs.

There is good observational evidence that all 19 objects are indeed binaries. The orbital elements themselves have been determined for 13 of them. For U Mon, LR Sco and IRAS 101745704 the radial velocity variability is linked to binary motion but the full orbital elements have not yet been quantified.

For some sources, a photometric time series can constrain the orbital period as well. In these objects the total line-ofsight extinction is varying with the orbital motion of the primary and this induces a variable line-of-sight reddening, in phase with the orbital period. This method has been widely applied to this type of source (e.g. Evans 1985; Waelkens et al. 1987; Van Winckel et al. 1999; Maas et al. 2003; Kiss et al. 2007) and is employed here to establish the likely orbital period of AR Pup, RV Tau and IW Car. Since this photometric phenomenon was discovered for RV Tauri stars and labelled the "RVb phenomenon", we use the same terminology, irrespective of whether the object is really an RV Tauri pulsator. We list whether this phenomenon was detected in a lightcurve in Table 1. It is an important qualifier for a system, because it strongly constrains the inclination of the circumstellar structure: when the "RVb phenomenon" is observed, the system must be seen under an inclination such that the line-of-sight grazes the circumbinary disk and the line-of-sight reddening changes during the orbital motion.

\section{The data}

\subsection{Interferometric data}

We do not list the detailed log of every observation, but graphically present the UV coverage for every source in Sect. 5 along with the results of our model fitting.

For a detailed account of the data reduction and calibration strategy, we refer to Menu et al. (2015). In short, the data 
Table 1. Binary post-AGB stars in our interferometric survey.

\begin{tabular}{|c|c|c|c|c|c|c|c|c|c|c|}
\hline $\mathrm{Nr}$. & IRAS & Name & $\begin{array}{c}\alpha_{2000} \\
{[\mathrm{~h} \mathrm{~m} \mathrm{~s}]}\end{array}$ & $\begin{array}{c}\delta_{2000} \\
{\left[o,{ }^{o},\right.}\end{array}$ & $\begin{array}{l}\text { Spectral } \\
\text { type }\end{array}$ & $\begin{array}{l}P_{\text {binary }} \\
\text { [days] }\end{array}$ & $\begin{array}{c}a_{1} \sin (i) \\
{[\mathrm{AU}]}\end{array}$ & $\begin{array}{l}f(M) \\
{\left[M_{\odot}\right]}\end{array}$ & $\mathrm{RVb}$ & Ref. \\
\hline 1 & $04440+2605$ & RV Tau & 044706.7 & +261045.6 & K3pv & $1180 \pm 15$ & & & $\mathrm{y}$ & Zsoldos (1996) \\
\hline 2 & $07008+1050$ & HD 52961 & 070339.6 & +104613.1 & F6I & $1297 \pm 7$ & 1.54 & 0.29 & $\mathrm{y}$ & Van Winckel et al. (1999) \\
\hline 3 & 07284-0940 & U Mon & 073047.5 & -094636.8 & K0IIb & $\sim 2600$ & & & $\mathrm{y}$ & Pollard et al. (2006) \\
\hline 4 & 08011-3627 & AR Pup & 080301.6 & -363547.9 & FOI & $1250 \pm 300$ & & & $\mathrm{y}$ & Kiss et al. (2007) \\
\hline 5 & 08544-4431 & & 085614.2 & -444310.7 & F3 & $508 \pm 2$ & 0.39 & 0.03 & - & Maas et al. (2003) \\
\hline 6 & 09256-6324 & IW Car & 092653.3 & -633748.9 & F7I & $\sim 1440$ & & & $\mathrm{y}$ & Pollard et al. (1996) \\
\hline 7 & $10158-2844$ & HR 4049 & 101807.6 & -285931.2 & $\mathrm{~A} 4 \mathrm{Ib} / \mathrm{II}$ & $434 \pm 1$ & 0.61 & 0.16 & $\mathrm{v}$ & Waelkens et al. (1991a) \\
\hline 8 & 10174-5704 & & 101916.9 & -571926.0 & - & $323 \pm 50$ & 0.17 & 0.007 & - & Maas (2003) \\
\hline 9 & $10456-5712$ & HD 93662 & 104738.4 & -572802.7 & K5 & $572 \pm 6$ & 0.015 & $1.4 \times 10^{-6}$ & - & Maas (2003) \\
\hline 10 & $11385-5517$ & HD 101584 & 114058.8 & -553425.8 & A6Ia & $218 \pm 1^{a}$ & & & $\mathrm{y}$ & Bakker et al. (1996) \\
\hline 11 & $12185-4856$ & SX Cen & 122112.6 & -491241.1 & G3V & $592 \pm 13$ & 1.23 & 0.70 & $\mathrm{y}$ & Deroo et al. (2006) \\
\hline 12 & $12222-4652$ & HD 108015 & 122453.5 & $-470907.5 \mathrm{v}$ & $\mathrm{F} 4 \mathrm{Ib} / \mathrm{II}$ & $914 \pm 4$ & 0.29 & 0.004 & - & van Winckel et al. (2009) \\
\hline 13 & $15469-5311$ & & 155043.8 & -532043.3 & F3 & 390 & 0.42 & 0.07 & - & van Winckel et al. (2009) \\
\hline 14 & $17038-4815$ & & 170736.6 & -481908.6 & G2p & $1386 \pm 12$ & 1.5 & 0.22 & $\mathrm{y}$ & Manick et al. (2017) \\
\hline 15 & $17243-4348$ & LR Sco & 172753.6 & -435046.3 & $\mathrm{G} 2$ & $\sim 475$ & & & - & Maas (2003) \\
\hline 16 & $17534+2603$ & $89 \mathrm{Her}$ & 175525.2 & +260300.0 & F3 & $288 \pm 1$ & 0.0 & $8 \times 10^{-4}$ & - & Waters et al. (1993) \\
\hline 17 & $18281+2149$ & ACHer & 183016.2 & +215200.6 & F2Iep & $1194 \pm 6$ & 1.39 & 0.25 & - & Van Winckel et al. (1998) \\
\hline 18 & $19125+0343$ & & 191501.2 & +034842.7 & $\mathrm{~F} 2$ & $520 \pm 2$ & 0.58 & 0.097 & - & van Winckel et al. (2009) \\
\hline 19 & 22327-1731 & HD 213985 & 223527.5 & -171526.9 & A0III & $258.6 \pm 0.3$ & 0.79 & 0.97 & $\mathrm{y}$ & Waelkens et al. (1995) \\
\hline
\end{tabular}

Notes. We give the coordinates together with the spectral type retrieved from Simbad. Most objects are confirmed binaries and some orbital parameters (orbital period, semi-major axis $a \sin (i)$, and mass function $f(M)$ ) are provided. We note for which targets the photometric "RVb" phenomenon has been reported. ${ }^{(a)}$ Díaz et al. (2007) reported a period of $144 \mathrm{~d}$ on an IAU symposium.

processing is done in two steps: 1) an extraction of raw correlated and total fluxes from the recorded data frames, and 2) the calibration of these raw fluxes into the science-worthy observables. The first data reduction step is performed with the 2.0 version of the Expert Work Station (EWS) software (Jaffe 2004), using a Python wrapper to extract observation by observation of both science and calibrator targets. The second step consists of determining the wavelength-dependent interferometric transfer function to calibrate the science observations from the interferometer's response to a point source. Science targets are observed in between calibrators to estimate the transfer function, which is subsequently interpolated to the times of the science observations.

In this paper we make use of the absolute correlated fluxes instead of the classically used visibilities. The visibility is equal to the correlated flux normalized with the zero-baseline (i.e. total) flux. Operating in the mid-IR, the zero-baseline flux measurements with MIDI are limited by the background subtraction, unlike the correlated flux measurements because of the way MIDI is designed to record the mid-IR interferogram (for more details, see Menu et al. 2015). Hence, if the calibration is able to capture the effective transfer function that corresponds to the science measurement, then correlated fluxes are preferred over visibilities. In contrast, the calibration of visibilities is principally more robust because effects that affect correlated and zerobaseline fluxes equally are divided away. Generally, we believe this is the case for our sample. For a few measurements we have doubts about the reliability of the transfer function estimation, and these are not included in our further analysis. For the estimation of the zero-baseline flux we combine the available MIDI fluxes (if reliable) with external measurements already published in the literature.

Although MIDI is a spectro-interferometric instrument, originally designed for studies of the amorphous and crystalline $\mathrm{N}$-band dust features, in this paper we focus on a single wavelength in-between the peaks of these features, $10.7 \mu \mathrm{m}$. The calibration step makes that the data are correlated in wavelength (e.g., due to seeing, etc.). Taking these correlations into account would complicate our analysis significantly, with limited gain. Moreover, at the long wavelength end of the $N$ band the measured fluxes become increasingly unreliable due to the increase in background noise. We choose the same reference wavelength of $10.7 \mu \mathrm{m}$ as in the interferometric surveys already mentioned previously (Menu et al. 2015; Boley et al. 2013; Monnier et al. 2009). This allows us to compare our results to these YSO disk samples. Our correlated fluxes are the weighted average of the measured fluxes between 10.55 and $10.85 \mu \mathrm{m}$, including only the error term due to photon noise in the weights. For the total error, the resulting photon noise term is added in quadrature with the error due to the calibration.

\subsection{Additional mid-IR spectra}

Infrared spectra of most sources were discussed with the focus on the solid state band profiles as probes for the dust grain processing (Gielen et al. 2007, 2009, 2011). We re-use these data here to define the slope of the continuum in the $N$-band outside the silicate feature. For these spectra an error of $15 \%$ on the absolute flux calibrations is assumed. Two of our sources, RV Tau and U Mon, were not included in the sample of Gielen et al. (2011). For UMon, we therefore also include the mid-IR fluxes measured by Lagadec et al. (2011) in our analysis (see Sect. 3.3). These fluxes were extracted from unresolved, but diffractionlimited, images in three narrowband filters (centred at 8.59, 11.85 , and $12.81 \mu \mathrm{m}$ ) with the VISIR instrument (Lagage et al. 2004) on the Very Large Telescope.

\subsection{Mid-IR variability}

Many of our targets show pulsational variability, often with very high amplitudes. In this section we consider whether some of the temporal variation in the MIDI spectra may be related to the stellar pulsation, and hence affect the interpretation of the 

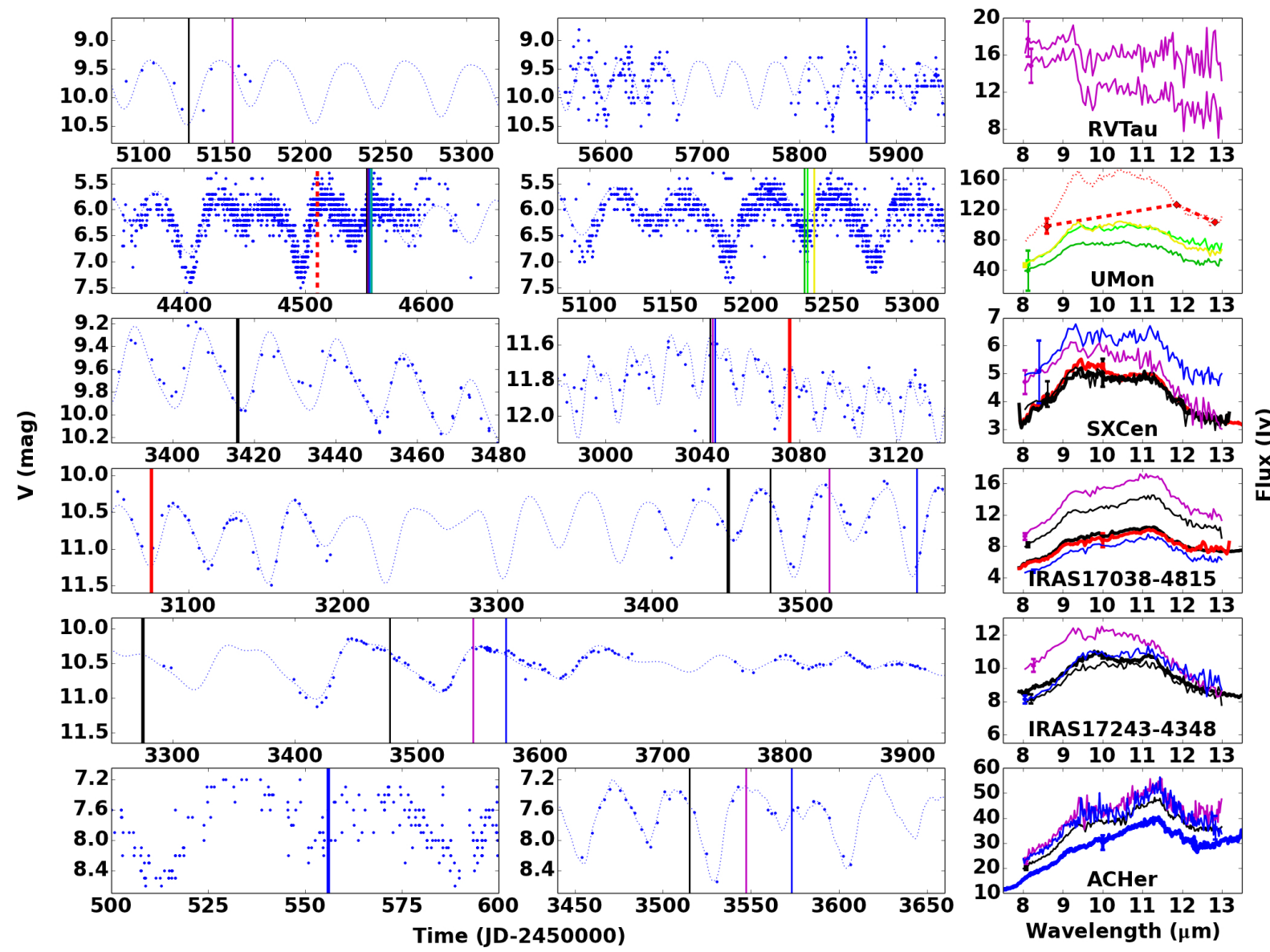

Fig. 1. Left panels: AAVSO and ASAS $V$ band light curves of the six stars with pulsation-related flux variations larger than 0.5 mag. The blue dotted line is a fit to the full light curve as described in Sect. 3.3, only shown to guide the eye. The coloured vertical lines indicate the time of observation of the mid-IR spectra shown in the right panels. Thick black, thick blue and thick red lines represent data from Spitzer, ISO and Timmi2, respectively. The thick red dashed line indicates the three-filter VISIR photometry of U Mon (published by Lagadec et al. 2011). The thin coloured lines represent our MIDI measurements at various epochs. Right panels: measured mid-IR fluxes for the indicated epochs in the left panels. The minimum error over the shown wavelength range is indicated for each spectrum with a bar. For RV Tau and U Mon, a few very noisy MIDI spectra are not included in the right panel. The red dotted line in the right panel of U Mon is the orange spectrum scaled to the VISIR fluxes.

interferometric observables. Not much is known about variability in the mid-IR of post-AGB disk sources, except the claims for a sine-like variation in the $10 \mu \mathrm{m}$ fluxes of AC Her by Gehrz (1971) and Shenton et al. (1992). Table 2 lists the pulsation properties of all our sample stars. We list whether the star is identified as a pulsator, and whether the pulsation is of RV Tau type. Objects for which no pulsation period is known in the literature are listed as pAGB. We also give typical peak-to-peak amplitudes of $V$ band brightness variations that are attributed to pulsations. Brightness variations of the RVb type are not taken into account here, since we attribute these to variable line-of-sight extinction during binary motion. Intrinsic mid-IR variability is more likely the result of a coupling between the stellar luminosity variation and the inner disk emissivity.

We identify six targets in our sample with amplitudes in the visual light curve larger than $0.5 \mathrm{mag}$ ( $>35 \%$ in flux variation). These are the candidates for which variable mid-IR emission may be detectable in the MIDI data, in response to the photospheric variations of the central luminous star. To check this hypothesis, we downloaded the light curves for these sources, from the ASAS catalogue (Pojmanski 2003; Pojmanski \& Maciejewski 2004; Pojmanski et al. 2005) if available, and otherwise from the AAVSO. AAVSO data are only used for the final MIDI epoch of RV Tau, all epochs of U Mon and the ISO epoch of AC Her. In all other cases we prefer the ASAS data due to their superior quality.

Figure 1 shows the resulting light curves, with the epochs of our mid-IR observations indicated by coloured vertical lines. To guide the eye, we include a fit to each of the visual light curves with frequencies determined from a Fourier analysis. The midIR spectra corresponding to these epochs are shown in the right panels, using the same colour and line-style coding. For each spectrum, the error is indicated at the wavelength with the smallest error in the $8-13 \mu \mathrm{m}$ wavelength range.

A close inspection of Fig. 1 shows that the epochs of observations are not in extrema of the lightcurve for most stars, except for U Mon and IRAS 17038-4815. For U Mon, the VISIR fluxes were taken near a pulsation maximum, and are significantly larger than the high-quality MIDI spectra obtained at a secondary minimum. That affects the analysis of the MIDI correlated fluxes (see Sect. 5). The clearest case is IRAS 170384815 , however. For this source the quality of the MIDI spectra is very good and the temporal sampling is favourable (three nearminimum phases with significantly lower mid-IR fluxes than the two near-maximum phases).

In summary, for the remainder of this paper we consider the mid-IR fluxes as intrinsically constant, except for UMon and 
Table 2. Pulsation properties of the sample stars.

\begin{tabular}{rccccc}
\hline \hline Nr. & IRAS & Type & $\Delta V_{\text {puls }}{ }^{a}$ & $P_{\text {pulsation }}{ }^{b}$ & Ref. \\
\hline 1 & $04440+2605$ & RVTau & 1.2 & 78.7 & 1,2 \\
2 & $07008+1050$ & Puls. & 0.15 & $72 \pm 3$ & 3 \\
3 & $07284-0940$ & RVTau & 1.1 & $92 \pm 3$ & 4,5 \\
4 & $08011-3627$ & RVTau & 0.5 & $76 \pm 4$ & 4 \\
5 & $08544-4431$ & Puls. & 0.15 & $\sim 72$ & 4,6 \\
6 & $09256-6324$ & Puls. & 0.45 & $72 \pm 1$ & 4 \\
7 & $10158-2844$ & pAGB & $<0.25$ & - & 7 \\
8 & $10174-5704$ & pAGB & $<0.10$ & - & 4 \\
9 & $10456-5712$ & pAGB & $<0.25$ & - & 4 \\
10 & $11385-5517$ & pAGB & $<0.10$ & - & 8 \\
11 & $12185-4856$ & RVTau & 0.6 & 32.8642 & 9 \\
12 & $12222-4652$ & Puls. & 0.15 & $\sim 60$ & 4,10 \\
13 & $15469-5311$ & Puls. & 0.15 & $\sim 50$ & 4 \\
14 & $17038-4815$ & RVTau & 1.50 & $37.9 \pm 1.5$ & 11 \\
15 & $17243-4348$ & RVTau & 0.8 & $100 \pm 4$ & 4,12 \\
16 & $17534+2603$ & Puls. & $<0.25$ & $65 \pm 3$ & 13,14 \\
17 & $18281+2149$ & RVTau & 1.8 & $75 \pm 2$ & 10 \\
18 & $19125+0343$ & pAGB & $<0.15$ & - & 4 \\
19 & $22327-1731$ & pAGB & $<0.05$ & - & 4 \\
\hline
\end{tabular}

Notes. Objects in which the characteristic RV Tau features have been identified in the light curve are listed as RV Tau. Otherwise, if a pulsation period has been detected it is listed as "Puls.", and if no period is known in the literature the source is denoted as "pAGB". The (maximum) amplitude of pulsational variability in the $V$ band is given, as well as the main pulsation period wherever relevant. ${ }^{(a)}$ Peak-to-peak amplitude. ${ }^{(b)}$ The main pulsation period.

References. (1) Taranova et al. (2009). (2) Zsoldos (1996). (3) Waelkens et al. (1991b). (4) Kiss et al. (2007). (5) Percy \& Bakos (1998). (6) Maas et al. (2003). (7) Waelkens et al. (1991a). (8) Bakker et al. (1996). (9) Shenton et al. (1994). (10) Samus et al. (2009). (11) Manick et al. (2017). ${ }^{(12)}$ Maas (2003). ${ }^{(13)}$ Dubath et al. (2011). ${ }^{(14)}$ Percy et al. (2000).

IRAS 17038-4815, which warrant a separate analysis at the different pulsation epochs.

\section{SED fitting}

Our interferometric model fitting in the next section requires two input parameters, the stellar effective temperature $T_{\text {eff }}$ and the stellar angular diameter $\theta_{\star}$ (Eq. (5.1)). Here we explain how we estimate both parameters. In addition, the results in this section allow us to estimate the stellar contribution to the $N$ band emission.

Since there are no published angular diameter measurements available for the sources in our sample, we resort to a uniform fitting of the stellar part of the SED. The fit parameters that determine a fully scaled stellar SED model at a single time are the effective temperature $T_{\text {eff }}$, the gravity $\log g$, the angular diameter $\theta_{\star}$, the reddening $E(B-V)$, and the metallicity $[\mathrm{Fe} / \mathrm{H}]$. We use Kurucz model atmospheres since the effective temperatures of our stars are generally above $3500 \mathrm{~K}$. For one star, IRAS 101745704, this lower bound is reached in the fitting, but we do not attempt a more detailed analysis as there is not sufficient photometry available for this source.

We keep the total reddening as a free parameter and assume that a single reddening law (the one of Fitzpatrick 2004) can represent the interstellar as well as the circumstellar contribution, the latter is expected to be particularly important for the $\mathrm{RVb}$ sources. We fix the metallicities based on the values listed in de Ruyter et al. (2006) and take the closest value available in our standard grid of Kurucz model atmospheres, or assume a solar metallicity if no spectroscopic determination is available.

The $T_{\text {eff }}, \log g, \theta_{\star}$ and $E(B-V)$ are fitted with a gridbased method, as described in Degroote et al. (2011). A reddened model is integrated over the observed photometric passbands before scaling it to the measured fluxes by adapting the angular diameter. Confidence intervals are determined using a $\chi^{2}$ statistic with four degrees of freedom (95\% probability or $2 \sigma)$.

Our strategy differs depending on the significance of the pulsation amplitude, relative to our ability to make a proper pulsation phase attribution. For the sources with an amplitude $V<0.5 \mathrm{mag}$, we include all reliable photometry in a single fit.

For the strong pulsators we collect photometry for a maximum and a minimum phase, and fit each set independently. For the RVb sources we try to collect both photometric sets during a long-term maximum phase, because this long-term variability is not caused by variations in the stellar properties.

We collect various photometric measurements from the literature (only for the pulsating sources) and from assorted on-line data catalogues. The sources of the photometry that is included in our analysis, are listed in Appendix A. We carefully select the photometric passbands to be included in our fitting process, the details of which are given in Appendix A.

Only measurements with effective wavelengths up to the $J$ (and in specific cases $H$ ) band were included in the fitting process. We avoid these passbands if there is an indication that there is already an excess at the respective wavelengths, but prefer to include them whenever possible.

Figures 2 and 3 show the SEDs of a single illustrative static and variable source, respectively. Also included are the confidence intervals of the $T_{\text {eff }}$ versus the $\theta_{\star}$ and $E(B-V)$, respectively. In Appendix B similar figures (Figs. B.1 and B.2) are included for all the other sources. The confidence intervals for $\log g$ generally remain unconstrained to the range (3.0; $0.0)$. For large-amplitude pulsators, there are two caveats. First, due to the scarcity of the used photometry, any systematic error on a subset has a direct impact on the results. In particular, the lack of reliable near-IR data is troublesome in certain cases (e.g. IRAS 17038-4815). Second, our method assumes that a sequence of hydrostatic Kurucz models can represent the highly dynamic atmosphere of these stars.

All sources except IRAS 10174-5704 and IRAS 10456-5712 have a spectroscopic temperature determination (de Ruyter et al. 2006). Taking the pulsation phase into account, we use these spectroscopic temperatures as an a posteriori constraint (included as the vertical red lines in the middle and right panels of Figs. 2 and 3). The $2 \sigma$ errors on the spectroscopic temperatures are uniformly assumed to be $10 \%$ for the static sources and $15 \%$ for the strong pulsators. The final parameter values, as listed in Table 3, are determined by taking the median value of the confidence intervals that remain after removing the points that do not agree with the spectroscopic temperature constraints. The final $2 \sigma$ errors are determined similarly, by taking the minimum and maximum value instead of the median.

In this SED analysis we neglect any scattered light contribution. For at least one source, 89 Her, this condition is severely violated, as was found by Hillen et al. (2013) and Hillen et al. (2014). The very small reddening that we find for a few sources (e.g. for IRAS $07008+1050$ it is smaller than the interstellar reddening in the Galactic extinction maps of Schlegel et al. 1998 and Drimmel \& Spergel 2001), might also be an indication for a non-negligible scattered-light flux fraction. Without resolved observations at near-IR or optical wavelengths, there is no general 

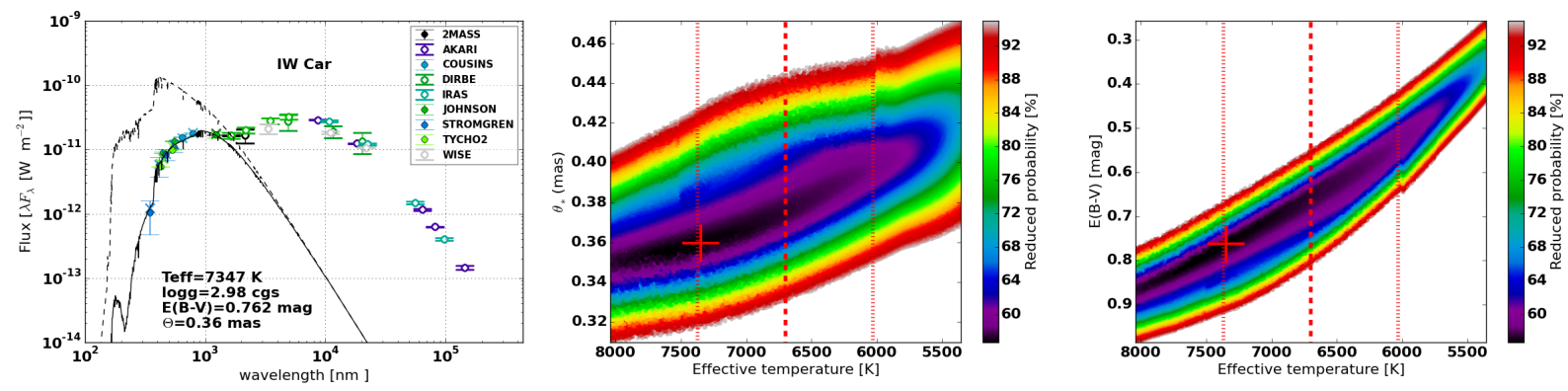

Fig. 2. An example (IW Car) of an SED of a post-AGB star with negligible pulsational variability. Left panel: measured photometry (circles), which are filled if the data were used in the fitting process. The colour indicates the photometric system, as listed in the legend. The full black line is the best-fit SED model that abides the spectroscopic temperature constraint (see the red cross in the middle and right panels). The exact parameter values of this model are given in the figure. The dashed black line is the same model, but unreddened. A consistent vertical scale, and colour coding for the photometric systems, is used for all stars in the sample (see Appendix B). Middle panel: confidence interval of the angular diameter $\theta_{\star}$ vs. the effective temperature $T_{\text {eff }}$. The dashed red line corresponds to the spectroscopically measured effective temperature. The dotted red lines indicate the $3 \sigma$ lower and upper limit on this temperature. Right panel: confidence interval of the reddening $E(B-V)$ vs. the effective temperature $T_{\text {eff }}$.
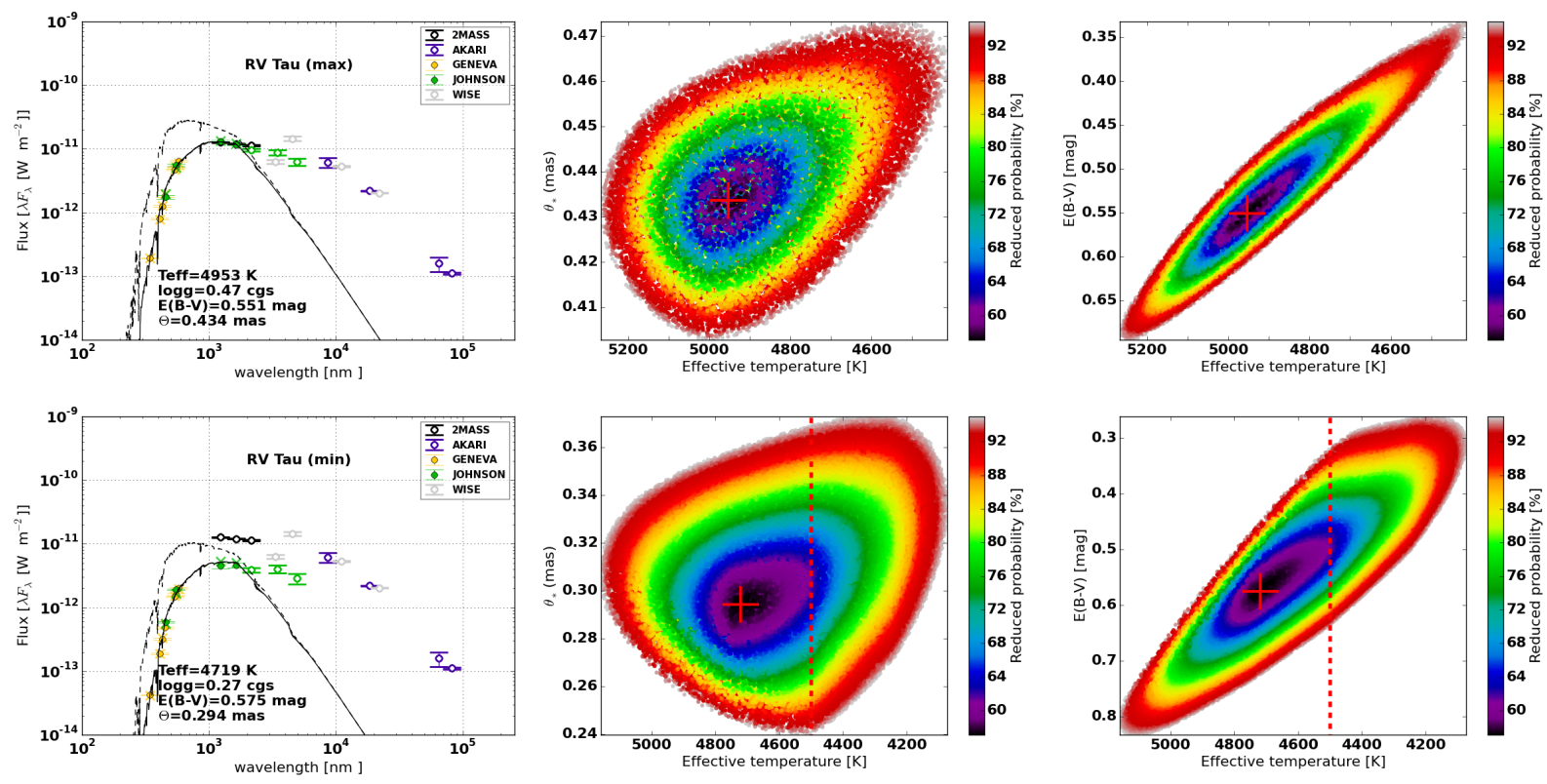

Fig. 3. Same as Fig. 2 but for a post-AGB star with strong pulsational variability (RV Tau itself). The upper and lower panels show optical and near-IR photometry at visual maximum and minimum pulsation phases, respectively. For visual reference we also include photometry for which no proper phase attribution can be made (mainly Tycho-2, 2MASS, and all mid- and far-IR data). In some cases these data may correspond to a different $\mathrm{RVb}$ phase.

way of estimating this scattered light contribution. Our angular diameters are thus likely overestimated. Fortunately, the sublimation radius only depends linearly on the angular diameter (see Eq. (5.1)), but quadratically on the effective temperature (which is spectroscopically constrained, hence less affected by scattering).

Finally, we note that our SEDs confirm the presence of variability in the mid-IR for U Mon and IRAS 17038-4815, as attested by the large scatter in the mid-IR fluxes as compared to the static sources. Similarly, it seems likely that RV Tauri and AC Her are intrinsically variable in the mid-IR as well, in contrast to the other stars in our category of "pulsators". This is consistent with the difference in visual pulsation amplitude between these objects.

The SEDs of our sources show a diversity of total infrared excesses, but near $10 \mu \mathrm{m}$ the dust always contributes an order of magnitude more flux than the photosphere.

\section{Interferometric model fitting}

\subsection{The models}

Although our sample is significant in number of stars, the uvcoverage per source is limited, in particular in terms of baseline position angle. For the current global analysis, we opt for a parametric approach. We make use of two parametric models to fit the mid-IR intensity distribution on the sky in 1D. For a large majority of our sources the baseline position angle coverage does not allow the detection of variations in the intensity distribution caused by inclination effects (for a more extensive discussion on this topic, see Menu et al. 2015). The effect of the inclination will be included in our sample-wise comparison with radiative transfer models (Sect. 8).

First we fit our data with the simplest possible model: a uniform disk. This has only the outer angular diameter $\Theta$ and the 
Table 3. Stellar parameters resulting from the SED fitting.

\begin{tabular}{|c|c|c|c|c|c|}
\hline $\mathrm{Nr}$. & IRAS & $\begin{array}{c}E(B-V)_{-2 \sigma}^{+2 \sigma} \\
(\mathrm{mag})\end{array}$ & $\begin{array}{c}T_{\text {eff }}+2 \sigma \\
(\mathrm{K})\end{array}$ & $\begin{array}{l}\theta_{\star} \begin{array}{l}+2 \sigma \\
-2 \sigma\end{array} \\
\text { (mas) }\end{array}$ & $\begin{array}{l}{[\mathrm{Fe} / \mathrm{H}]} \\
(\mathrm{dex})\end{array}$ \\
\hline \multirow[t]{2}{*}{1} & $04440+2605$ & $0.5_{-0.2}^{+0.2}$ & $4875_{-450}^{+400}$ & $0.44_{-0.04}^{+0.03}$ & -0.5 \\
\hline & & $0.5_{-0.3}^{+0.3}$ & $4550_{-475}^{+600}$ & $0.31_{-0.07}^{+0.06}$ & -0.5 \\
\hline 2 & $07008+1050$ & $0.04_{-0.04}^{+0.09}$ & $6050_{-225}^{+400}$ & $0.270_{-0.010}^{+0.014}$ & -4.0 \\
\hline \multirow[t]{2}{*}{3} & 07284-0940 & $0.18_{-0.18}^{+0.3}$ & $5050_{-400}^{+450}$ & $1.2_{-0.2}^{+0.2}$ & -1.0 \\
\hline & & $0.16_{-0.16}^{+0.2}$ & $5025_{-300}^{+350}$ & $0.58_{-0.10}^{+0.10}$ & -1.0 \\
\hline \multirow[t]{2}{*}{4} & 08011-3627 & $0.4_{-0.4}^{+0.2}$ & $5925_{-725}^{+1250}$ & $0.22_{-0.05}^{+0.03}$ & -1.0 \\
\hline & & $0.2_{-0.2}^{+0.3}$ & $5875_{-775}^{+1025}$ & $0.14_{-0.02}^{+0.02}$ & -1.0 \\
\hline 5 & $08544-4431$ & $1.40_{-0.2}^{+0.19}$ & $7225_{-700}^{+750}$ & $0.54_{-0.08}^{+0.09}$ & -0.5 \\
\hline 6 & 09256-6324 & $0.7_{-0.3}^{+0.2}$ & $6700_{-650}^{+675}$ & $0.38_{-0.07}^{+0.07}$ & -1.0 \\
\hline 7 & $10158-2844$ & $0.21_{-0.16}^{+0.09}$ & $7750_{-975}^{+525}$ & $0.49_{-0.06}^{+0.07}$ & -4.0 \\
\hline 8 & $10174-5704$ & $0.88_{-0.18}^{+0.2}$ & $3500_{-175}^{+175}$ & $1.09_{-0.05}^{+0.06}$ & $0.0^{b}$ \\
\hline 9 & $10456-5712$ & $0.34_{-0.34}^{+0.3}$ & $4275_{-550}^{+600}$ & $1.8_{-0.3}^{+0.4}$ & $0.0^{b}$ \\
\hline 10 & $11385-5517$ & $0.35_{-0.15}^{+0.11}$ & $8350_{-700}^{+1000}$ & $0.26_{-0.03}^{+0.03}$ & $0.0^{c}$ \\
\hline \multirow[t]{2}{*}{11} & $12185-4856$ & $0.3_{-0.3}^{+0.2}$ & $5925_{-625}^{+1225}$ & $0.18_{-0.03}^{+0.02}$ & -1.0 \\
\hline & & $0.3_{-0.3}^{+0.3}$ & $5850_{-1050}^{+1350}$ & $0.138_{-0.018}^{+0.03}$ & -1.0 \\
\hline 12 & $12222-4652$ & $0.17_{-0.12}^{+0.13}$ & $7000_{-575}^{+700}$ & $0.178_{-0.013}^{+0.010}$ & 0.0 \\
\hline 13 & $15469-5311$ & $1.34_{-0.2}^{+0.16}$ & $7425_{-675}^{+825}$ & $0.23_{-0.02}^{+0.03}$ & 0.0 \\
\hline \multirow[t]{2}{*}{14} & $17038-4815$ & $0.3_{-0.3}^{+0.2}$ & $4375_{-575}^{+775}$ & $0.29_{-0.07}^{+0.04}$ & -1.5 \\
\hline & & $0.7_{-0.3}^{+0.4}$ & $4575_{-525}^{+900}$ & $0.18_{-0.04}^{+0.04}$ & -1.5 \\
\hline \multirow[t]{2}{*}{15} & $17243-4348$ & $0.3_{-0.3}^{+0.3}$ & $5075_{-800}^{+825}$ & $0.16_{-0.02}^{+0.03}$ & 0.0 \\
\hline & & $0.3_{-0.2}^{+0.2}$ & $4850_{-400}^{+625}$ & $0.14_{-0.02}^{+0.03}$ & 0.0 \\
\hline 16 & $17534+2603$ & $0.07_{-0.07}^{+0.10}$ & $6850_{-325}^{+350}$ & $0.52_{-0.03}^{+0.03}$ & 0.0 \\
\hline \multirow[t]{2}{*}{17} & $18281+2149$ & $0.26_{-0.26}^{+0.17}$ & $6200_{-950}^{+700}$ & $0.39_{-0.05}^{+0.04}$ & -1.5 \\
\hline & & $0.03_{-0.03}^{+0.07}$ & $4800_{-150}^{+150}$ & $0.318_{-0.015}^{+0.014}$ & -1.5 \\
\hline 18 & $19125+0343$ & $0.79_{-0.07}^{+0.15}$ & $7275_{-300}^{+1250}$ & $0.140_{-0.011}^{+0.009}$ & -0.5 \\
\hline 19 & $22327-1731$ & $0.12_{-0.12}^{+0.14}$ & $8275_{-850}^{+800}$ & $0.085_{-0.013}^{+0.013}$ & -1.0 \\
\hline
\end{tabular}

Notes. The pulsating sources have two entries, one for a minimum and a maximum pulsation phase. Metallicities are taken from de Ruyter et al (2006) unless otherwise noted. ${ }^{(a)} \mathrm{H}$ band fluxes are also included in the fit for sources 1,8 and $17,{ }^{(b)}$ This metallicity is assumed. ${ }^{(c)}$ The spectroscopic constraints for this source come from Kipper (2005). ${ }^{(f)} \mathrm{We}$ are cautious about IRAS 10174-5704, given the limited data and the limiting $T_{\text {eff }}=3500 \mathrm{~K}$ in the atmosphere grid.

total integrated flux as free parameters. The advantage of this model is that it is widely used and allows to make a simple angular size estimate for each object in the sample.

Second, we use the semi-physical disk model applied by Menu et al. (2015) to a large sample of protoplanetary disks observed with MIDI. The specific intensity of this model is parameterized as a function of the angular radius $\rho$ and optical depth $\tau$ as follows:

$$
I_{v}(\rho)=(1-\exp (-\tau)) B_{v}(T(\rho)) \quad \text { for } \quad \rho_{\text {sub }}<\rho<\rho_{\text {out }},
$$

and zero elsewhere, with

$T(\rho)=T_{\text {sub }}\left(\frac{\rho}{\rho_{\text {sub }}}\right)^{-q}$.

Here, $T_{\text {sub }}$ and $\rho_{\text {sub }}$ are the sublimation temperature and angular sublimation radius of the dust. We keep the dust sublimation temperature fixed at $T_{\text {sub }}=1500 \mathrm{~K}$. We assume that the dust disk starts at sublimation radius. The angular sublimation radius

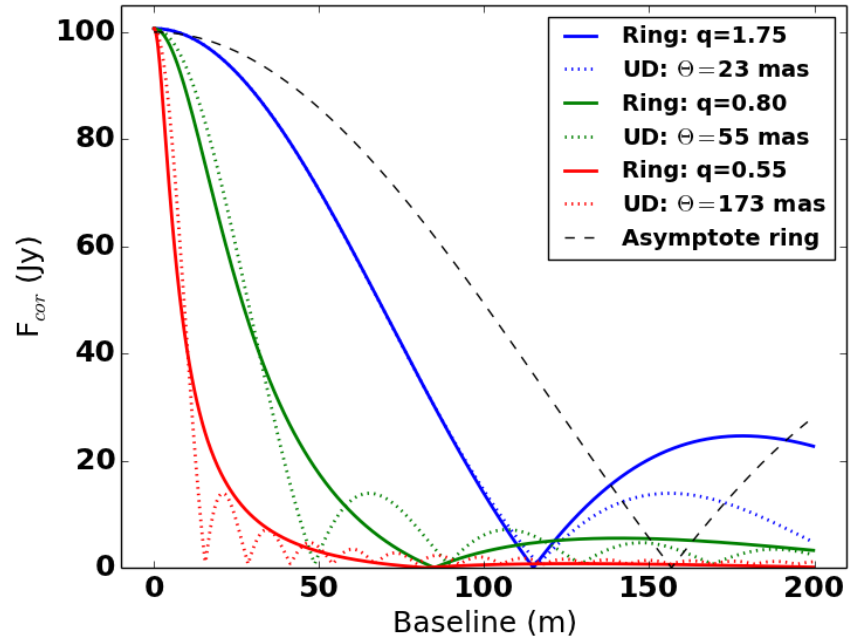

Fig. 4. Simulated model visibility curves. Three ring models with different power law values are shown as full lines (see the legend for the colour coding). The inner and outer ring radii are fixed to the values adopted for the $89 \mathrm{Her}$ system. The correlated flux at zero baseline (i.e., the total flux) is fixed at $100 \mathrm{Jy}$ for comparison purposes. For each ring model, the uniform disk model that crosses the correlated flux curve at $40 \mathrm{Jy}$ is shown for comparison. The black dashed line represents the asymptotic limit of the ring model: an infinitesimally thin ring at the sublimation radius.

is calculated as follows:

$\rho_{\text {sub }}=\frac{\theta_{\star}}{2}\left(\frac{T_{\text {eff }}}{T_{\text {sub }}}\right)^{2}$

(Dullemond \& Monnier 2010), with $\theta_{\star}$ and $T_{\text {eff }}$ the stellar angular diameter and effective temperature, respectively. We keep the outer radius fixed at $\rho_{\text {out }}=100 \rho_{\text {sub }}$ but our results are insensitive to the exact choice.

The correlated flux corresponding to a given baseline and wavelength is then computed as the 1D Hankel transform of the intensity distribution, integrated over the radial angular coordinate on the sky. The only free fit parameters in this model are the temperature gradient $q$ and the total integrated flux. The average optical depth is a derived parameter.

Figure 4 compares the correlated flux behaviour of our two models. The corresponding images for these models are displayed in Fig. 5. Two extreme cases of a large $(q=0.55)$ and a small $(q=1.75)$ ring are used, together with the median value in our sample ( $q=0.8$; see Sect. 5.2). The total flux $F_{\text {tot }}=100 \mathrm{Jy}$ and sublimation radius are kept fixed in all three models (using the stellar parameters of 89 Her). A uniform disk is shown that reaches a correlated flux of $40 \mathrm{Jy}$ at the same baseline length as in the corresponding ring model. Figure 4 shows that for correlated fluxes above $40 \%$ the two models are indistinguishable. Only at longer baselines the models behave significantly different. By applying both parametric models to our observations, we have a better diagnostic to analyse the sample properties.

Although the value of $q$ should not be over-interpreted, classical models of flaring protoplanetary disks give rise to temperature profiles of $T(\rho) \propto \rho^{-1 / 2}$ (Kenyon \& Hartmann 1987). The steepest profile one would expect for a passively irradiated disk is obtained in the geometrically thin limit, and corresponds to $T(\rho) \propto \rho^{-3 / 4}$ (Armitage 2010; Kenyon \& Hartmann 1987). For a spherical stellar wind, the expected temperature profile corresponds to $T(\rho) \propto \rho^{-2 / 5}$ (Lamers \& Cassinelli 1999). In reality, the radial optical depth profile is important as well, while it is 


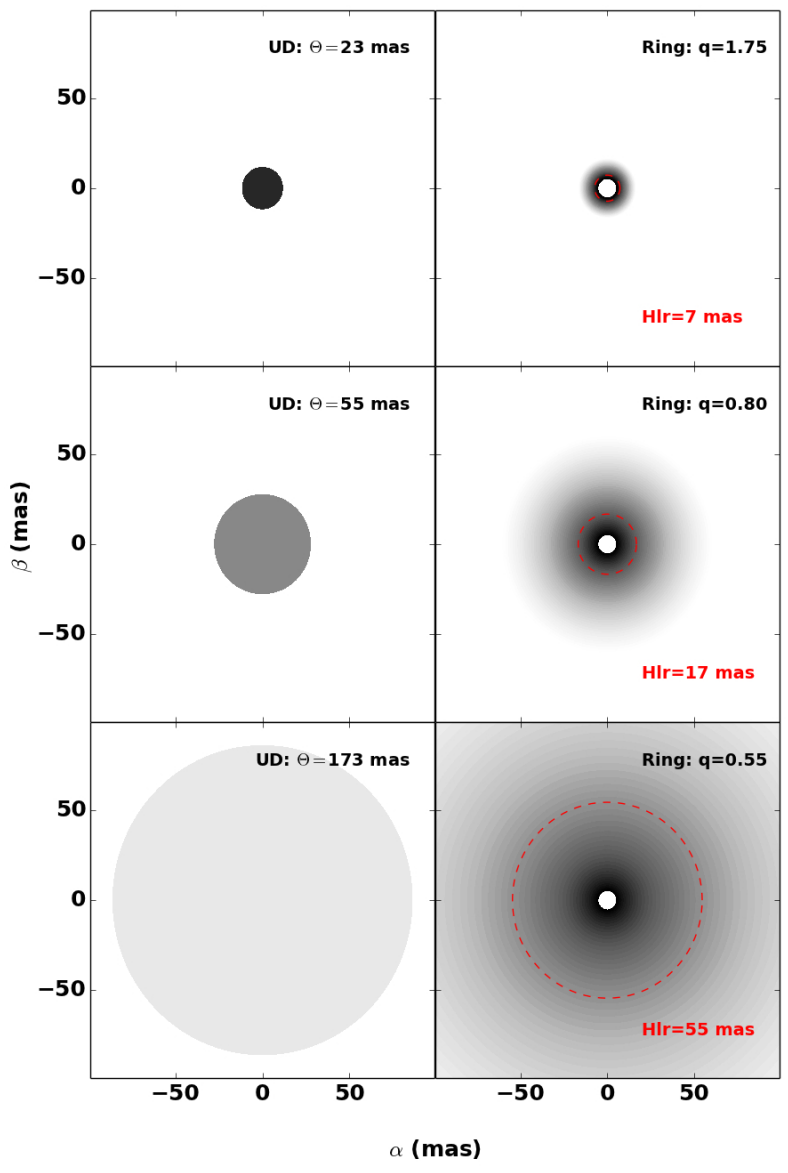

Fig. 5. Simulated model images corresponding to the visibility curves shown in Fig. 4. Left panels: uniform disks; right panels: the semiphysical ring models. A common intensity normalization is used across the panels. Also the same logarithmic greyscale is applied for all panels. The half-light radius for each of the ring models (see Sect. 7.1) is indicated by the red dashed circle. The field of view of the image is limited for clarity of presentation.

assumed to be constant here $(\tau<1)$. Our fitted value of $q$ can thus not be directly associated with the true temperature gradient in a given source, but merely acts as a parameter that distributes flux over subsequent radial annuli. Our model assumes the dust emission to start at the sublimation radius and to be continuous outwards. For sources where there is only dust further out, the fit will compensate the too small assumed inner radius by making $q$ smaller (i.e. a larger apparent structure, see Figs. 4 and 5).

One can define the apparent size of a given ring model with a single parameter, called the half-light radius $(h l r)$, which is computed from the following implicit equation (see Menu et al. 2015):

$\frac{F_{\mathrm{tot}, v}}{2}=\int_{\rho_{\mathrm{sub}}}^{h l r} \mathrm{~d} \rho 2 \pi \rho I_{v}(\rho)$.

The half-light radius represents the radius within which half of the mid-IR flux is emitted. The half-light radius of each model in Fig. 5 is indicated in red.

\subsection{The fit strategy}

We describe the fit strategy adopted in this work. Both our models have two fit parameters: $q$ or $\Theta$ and the total flux $F_{\text {tot }}$.
To locate best-fitting models and to estimate parameter uncertainties, the Markov chain Monte Carlo (MCMC) method is widely used. In this paper we use the emcee package implemented in Python (Foreman-Mackey et al. 2013). An MCMC allows to randomly draw realizations of the model parameters according to the Bayesian posterior probability distribution determined by the data, $P(M \mid D)$. Bayes' theorem states that this posterior probability distribution is proportional to the product of the prior probability of the model $P(M)$ with the likelihood $P(D \mid M)$. We assume uniform priors, allowing $F_{\text {tot }} \in(0,500) \mathrm{Jy}$, and $\Theta \in(0,500)$ mas (the upper limit being the field-of-view of MIDI). For the ring model we assume $q \in(0,2.5)$. The lower value corresponds to a uniform ring with the temperature equal to the sublimation temperature. The upper limit can be considered as the start of the asymptotic regime toward the infinitesimally thin ring (see Fig. 7). The likelihood is proportional to $\exp \left(-\chi^{2} / 2\right)$, which assumes Gaussian distributed measurement errors. The $\chi^{2}$ includes the MIDI fluxes, but also the additional fluxes collected for many sources (see Sect. 3.2). For each parameter we take the median, and the 16th and 84th percentile of the marginalized distribution as the final parameter value, and its lower and upper $1 \sigma$ error, respectively (see Table 4 ).

We fit $F_{\text {tot }}$, instead of fixing it to the average of all zerobaseline fluxes, because short-baseline correlated fluxes are also sensitive to this parameter.

Due to the limited uv-coverage per source, and the $\mathrm{S} / \mathrm{N}$-dependent super-resolution capability of an interferometer, we determine whether a source is partially resolved by means of a statistical criterion. We use the concept of Bayesian model selection, in which the evidence provided by the data is weighed against or in favour of a null hypothesis (Kass \& Raftery 1995). Our null hypothesis is defined as the object is unresolved, i.e. $\Theta=0$, versus the alternative hypothesis that the object is resolved, or $\Theta>0$. We compute the Bayes factor $B_{10}$ and reject the null hypothesis in favour of the alternative if $B_{10}>10$ (see Table 4). We fit the uniform disk model twice (with and without $\Theta=0$ ), and take the ratio of the Bayesian evidences (provided by emcee) as the Bayes factor. We do not use the ring model for deciding the resolvedness of each source because it incorporates a lower limit on the size (see Sect. 5.1).

\subsection{The fit results}

The interferometric fit results are graphically depicted in Fig. 6 for two sources in our sample, the remainder is included in Appendix B (Figs. B.3 to B.4). The quantified results are given in Table 4. The figures show the 2-dimensional uv-coverage, the correlated fluxes as a function of projected baseline length and $\chi^{2}$-maps for both models. One hundred realizations randomly drawn according to the posterior probability distribution that was derived with the MCMC are shown on top of the $\chi^{2}$-maps as well. The quantified results are given in Table 4 . We note that the half-light radii found for the minimum and maximum pulsation phase of U Mon and IRAS 17038-4815 are very similar (but the optical depths differ), even though the total flux increases by $\geq 50 \%$. This is not unexpected because the inner rim radius cannot change dramatically over the course of a pulsation cycle. However, the inner rim temperature, which we assume fixed, can respond almost instantaneously (Nagel et al. 2010).

All sources in our sample, except SX Cen (see also Deroo et al. 2006) and IRAS 22327-1731, are resolved. For IRAS 22327-1731 we only have one correlated flux measurement. Even though this flux is marginally smaller than the 
Table 4. Result of the interferometric model fitting.

\begin{tabular}{|c|c|c|c|c|c|c|c|c|c|c|}
\hline Index & IRAS & $\begin{array}{l}\Theta_{-1 \sigma}^{+1 \sigma} \\
\text { (mas) }\end{array}$ & $\begin{array}{l}F_{\text {tot }}+1 \sigma \\
(\mathrm{Jy})\end{array}$ & $\begin{array}{l}\rho_{\text {sub }}{ }_{-1 \sigma}^{+1 \sigma} \\
\text { (mas) }\end{array}$ & $q_{-1 \sigma}^{+1 \sigma}$ & $\begin{array}{l}F_{\text {tot }}+1 \sigma \\
(\mathrm{Jy})\end{array}$ & $\tau_{\mathrm{eff}}{ }_{-1 \sigma}^{+1 \sigma}$ & $\begin{array}{l}h l r_{-1 \sigma}^{+1 \sigma} \\
\text { (mas) }\end{array}$ & Bayes factor $B_{10}$ & Resolved? \\
\hline 1 & $04440+2605$ & $13.9_{-0.6}^{+0.6}$ & $11.8_{-0.8}^{+0.7}$ & $2.3_{-0.2}^{+0.2}$ & $1.11_{-0.05}^{+0.06}$ & $12_{-1}^{+1}$ & $0.27_{-0.02}^{+0.02}$ & $4.3_{-0.2}^{+0.2}$ & $10^{14}$ & $\mathrm{y}$ \\
\hline 2 & $07008+1050$ & $37_{-7}^{+6}$ & $3.9_{-0.5}^{+0.5}$ & $2.2_{-0.2}^{+0.1}$ & $0.69_{-0.06}^{+0.13}$ & $3.7_{-0.7}^{+0.6}$ & $0.02_{-0.01}^{+0.01}$ & $10_{-3}^{+3}$ & $\sim 16$ & $\mathrm{y}$ \\
\hline \multirow[t]{2}{*}{3} & 07284-0940 (max) & $50.0_{-0.5}^{+0.5}$ & $125_{-3}^{+3}$ & $7.0_{-0.9}^{+0.9}$ & $0.87_{-0.02}^{+0.02}$ & $157_{-7}^{+7}$ & $0.196_{-0.005}^{+0.005}$ & $18.4_{-0.6}^{+0.6}$ & $10^{233}$ & $\mathrm{y}$ \\
\hline & 07284-0940 (min) & $41.2_{-0.6}^{+0.7}$ & $75_{-3}^{+3}$ & $7.0_{-0.9}^{+0.9}$ & $0.97_{-0.04}^{+0.04}$ & $90_{-5}^{+5}$ & $0.15_{-0.01}^{+0.01}$ & $15.6_{-0.9}^{+0.9}$ & $10^{99}$ & $\mathrm{y}$ \\
\hline 4 & 08011-3627 & $63_{-1}^{+1}$ & $121_{-5}^{+6}$ & $1.7_{-0.4}^{+0.3}$ & $0.507_{-0.005}^{+0.005}$ & $121_{-5}^{+5}$ & $0.25_{-0.01}^{+0.01}$ & $25_{-1}^{+1}$ & $10^{52}$ & $\mathrm{y}$ \\
\hline 5 & $08544-4431$ & $46_{-2}^{+2}$ & $153_{-6}^{+4}$ & $6.2_{-0.8}^{+0.8}$ & $0.90_{-0.02}^{+0.02}$ & $171_{-5}^{+5}$ & $0.32_{-0.01}^{+0.01}$ & $14.8_{-0.4}^{+0.4}$ & $10^{271}$ & $\mathrm{y}$ \\
\hline 6 & 09256-6324 & $38_{-3}^{+3}$ & $102_{-3}^{+4}$ & $3.8_{-0.5}^{+0.5}$ & $0.86_{-0.03}^{+0.04}$ & $103_{-4}^{+4}$ & $0.42_{-0.04}^{+0.05}$ & $10.3_{-0.8}^{+0.8}$ & $10^{8}$ & $\mathrm{y}$ \\
\hline 7 & $10158-2844$ & $42_{-2}^{+2}$ & $23.4_{-0.4}^{+0.5}$ & $6.6_{-0.7}^{+0.9}$ & $1.09_{-0.04}^{+0.05}$ & $23.9_{-0.6}^{+0.6}$ & $0.062_{-0.005}^{+0.006}$ & $12.5_{-0.6}^{+0.6}$ & $10^{20}$ & $\mathrm{y}$ \\
\hline 8 & $10174-5704$ & $140_{-2}^{+1}$ & $53_{-2}^{+2}$ & $3.0_{-0.2}^{+0.1}$ & $0.457_{-0.004}^{+0.004}$ & $54_{-2}^{+2}$ & $0.018_{-0.001}^{+0.001}$ & $75_{-4}^{+4}$ & $10^{160}$ & $\mathrm{y}$ \\
\hline 9 & $10456-5712$ & $58_{-3}^{+3}$ & $163_{-7}^{+7}$ & $7.4_{-1.2}^{+1.2}$ & $0.92_{-0.04}^{+0.04}$ & $170_{-10}^{+10}$ & $0.23_{-0.02}^{+0.02}$ & $18_{-1}^{+1}$ & $10^{19}$ & $\mathrm{y}$ \\
\hline 10 & $11385-5517$ & $27.9_{-0.6}^{+0.6}$ & $80_{-2}^{+2}$ & $4.0_{-0.6}^{+0.4}$ & $1.00_{-0.02}^{+0.02}$ & $81_{-2}^{+2}$ & $0.45_{-0.02}^{+0.02}$ & $8.5_{-0.2}^{+0.2}$ & $10^{101}$ & $\mathrm{y}$ \\
\hline 11 & $12185-4856$ & $<27$ & $5.3_{-0.4}^{+0.3}$ & $1.4_{-0.3}^{+0.2}$ & $>0.75$ & $5.2_{-0.3}^{+0.4}$ & $>0.1$ & $<5.2$ & $\sim 0.1$ & $\mathrm{n}$ \\
\hline 12 & $12222-4652$ & $28_{-1}^{+1}$ & $31.9_{-0.8}^{+0.8}$ & $1.9_{-0.2}^{+0.2}$ & $0.72_{-0.01}^{+0.01}$ & $33_{-1}^{+1}$ & $0.29_{-0.01}^{+0.01}$ & $7.7_{-0.4}^{+0.34}$ & $10^{33}$ & $\mathrm{y}$ \\
\hline 13 & $15469-5311$ & $36_{-4}^{+1}$ & $40_{-2}^{+2}$ & $2.9_{-0.4}^{+0.2}$ & $0.77_{-0.05}^{+0.01}$ & $40_{-2}^{+2}$ & $0.20_{-0.04}^{+0.05}$ & $10_{-1}^{+2}$ & $10^{11}$ & $\mathrm{y}$ \\
\hline \multirow[t]{2}{*}{14} & $17038-4815(\max )$ & $19.8_{-0.9}^{+0.8}$ & $14.5_{-0.7}^{+0.7}$ & $1.2_{-0.2}^{+0.2}$ & $0.68_{-0.02}^{+0.02}$ & $14.6_{-0.8}^{+0.8}$ & $0.25_{-0.02}^{+0.02}$ & $5.8_{-0.4}^{+0.5}$ & $10^{18}$ & $\mathrm{y}$ \\
\hline & $17038-4815(\mathrm{~min})$ & $17_{-1}^{+1}$ & $9.1_{-0.8}^{+0.9}$ & $1.2_{-0.2}^{+0.2}$ & $0.72_{-0.03}^{+0.03}$ & $9.1_{-0.8}^{+0.9}$ & $0.20_{-0.02}^{+0.02}$ & $5.0_{-0.5}^{+0.5}$ & $10^{5}$ & $\mathrm{y}$ \\
\hline 15 & $17243-4348$ & $14.7_{-0.7}^{+0.8}$ & $10.3_{-0.6}^{+0.5}$ & $0.9_{-0.2}^{+0.2}$ & $0.70_{-0.02}^{+0.02}$ & $10.5_{-0.5}^{+0.5}$ & $0.35_{-0.03}^{+0.03}$ & $4.0_{-0.3}^{+0.3}$ & $10^{10}$ & $\mathrm{y}$ \\
\hline \multirow[t]{2}{*}{16} & $17534+2603$ & $22.2_{-0.5}^{+0.6}$ & $79_{-3}^{+3}$ & $5.4_{-0.3}^{+0.3}$ & $1.9_{-0.1}^{+0.1}$ & $79_{-3}^{+3}$ & $0.90_{-0.07}^{+0.08}$ & $7.0_{-0.2}^{+0.2}$ & $10^{51}$ & $\mathrm{y}$ \\
\hline & $17534+2603{\text { (alt })^{a}}^{a}$ & & & $3.9_{-0.3}^{+0.3}$ & $1.12_{-0.04}^{+0.04}$ & $83_{-4}^{+4}$ & $0.65_{-0.05}^{+0.05}$ & $7.2_{-0.3}^{+0.3}$ & & \\
\hline 17 & $18281+2149$ & $65.7_{-0.7}^{+0.7}$ & $42_{-2}^{+2}$ & $3.3_{-0.4}^{+0.5}$ & $0.537_{-0.009}^{+0.008}$ & $41_{-2}^{+2}$ & $0.030_{-0.003}^{+0.002}$ & $38_{-2}^{+3}$ & $10^{101}$ & $\mathrm{y}$ \\
\hline 18 & $19125+0343$ & $20.0_{-0.6}^{+0.5}$ & $25_{-1}^{+1}$ & $1.6_{-0.3}^{+0.1}$ & $0.74_{-0.01}^{+0.01}$ & $25_{-1}^{+1}$ & $0.33_{-0.02}^{+0.02}$ & $6.1_{-0.3}^{+0.3}$ & $10^{42}$ & $\mathrm{y}$ \\
\hline 19 & $22327-1731$ & $<20$ & $5.3_{-0.7}^{+0.6}$ & $1.3_{-0.2}^{+0.2}$ & $>0.67$ & $4.9_{-0.7}^{+0.8}$ & $>0.08$ & $<6.5$ & $\sim 1$ & $\mathrm{n}$ \\
\hline
\end{tabular}

Notes. The mid-IR variable sources identified in Sect. 3.3 have two entries, one for a minimum and a maximum pulsation phase. For unresolved sources we give $3 \sigma$ upper or lower limits on the determined size parameters, otherwise the uncertainties are at the $1 \sigma$ level. We define $\tau_{\text {eff }}$ as the scale factor $\left(\tau_{\text {eff }}=(1-\exp -\tau)\right.$. For small $\tau$, the effective scale factor reduces to $\tau .{ }^{(a)}$ The results of the fit when assuming the stellar parameters of Hillen et al. (2013), i.e., $T_{\mathrm{eff}}=6250 \mathrm{~K}$ and $\theta_{\star}=0.45$ mas.

two total flux measurements, statistically we must consider this source to be unresolved.

In the following section we discuss some peculiar individual objects in more detail.

\section{Notes on some individual targets}

\subsection{U Mon (nr. 3)}

The best-fit value of $F_{\text {tot }}$ is the same for the uniform disk and the ring model for almost all objects in the sample. For one source, U Mon, the fitted $F_{\text {tot }}$ are discrepant both for the data in minimum as in maximum light. It has the most extensive uv-coverage among the whole sample, in combination with very precise correlated flux measurements, but few total flux constraints. The data for this source were taken on several epochs, either corresponding to a maximum phase in the pulsation cycle or to a secondary minimum phase. At the maximum phase, the only total flux measurement is based on the VISIR fluxes that are extrapolated with the shape of the MIDI spectrum observed for the minimum phase (the red cross in Fig. B.3; see also Fig. 1). Formally, we assume a large error for this measurement since it is based on an extrapolation. We note, however, that the VISIR fluxes are reliable and significantly larger than the minimum phase MIDI flux at the same wavelengths (Sect. 3.3). A visual inspection of the fit to these data shows that the ring model fits the fluxes at all baselines, in contrast to the uniform disk model which underestimates the zero-baseline flux. The $\chi^{2}$-map shows that a uniform disk model with the same $F_{\text {tot }}$ as the ring model, fits the data significantly worse. This is due to the excellent sampling in baseline length at a single position angle, which puts precise constraints on the slope of the visibility curve.

At the minimum phase, this source has a large coverage in baseline position angle, compared to any other star in our sample. The distinct fluxes at the various $\sim 70 \mathrm{~m}$ baselines, which have different position angles, suggests a clear variation in extension of the object as a function of position angle. This cannot be accommodated by our 1D models, as is reflected in the atypically high $\chi^{2}$ values for this data set. Finally, we note that the same uv point corresponding to the $30 \mathrm{~m}$ baseline is probed at the maximum pulsation phase as well. The corresponding correlated fluxes are very different, thereby confirming our interpretation of the total flux variations. The pulsation-induced flux variations and the high-quality correlated fluxes, justify a more detailed analysis in a future paper.

\subsection{AR Pup (nr. 4)}

AR Pup is an RV Tauri pulsator and the only star in our sample for which the total infrared luminosity dominates over the dereddened optical fluxes (Fig. B.2). This is indicative that we see the disk close to edge-on (de Ruyter et al. 2006). As the disk is optically thick in the radial direction, the visible component of the SED is likely caused by scattering only, with no direct light being observed. This RV Tauri pulsator has a variable mean magnitude 

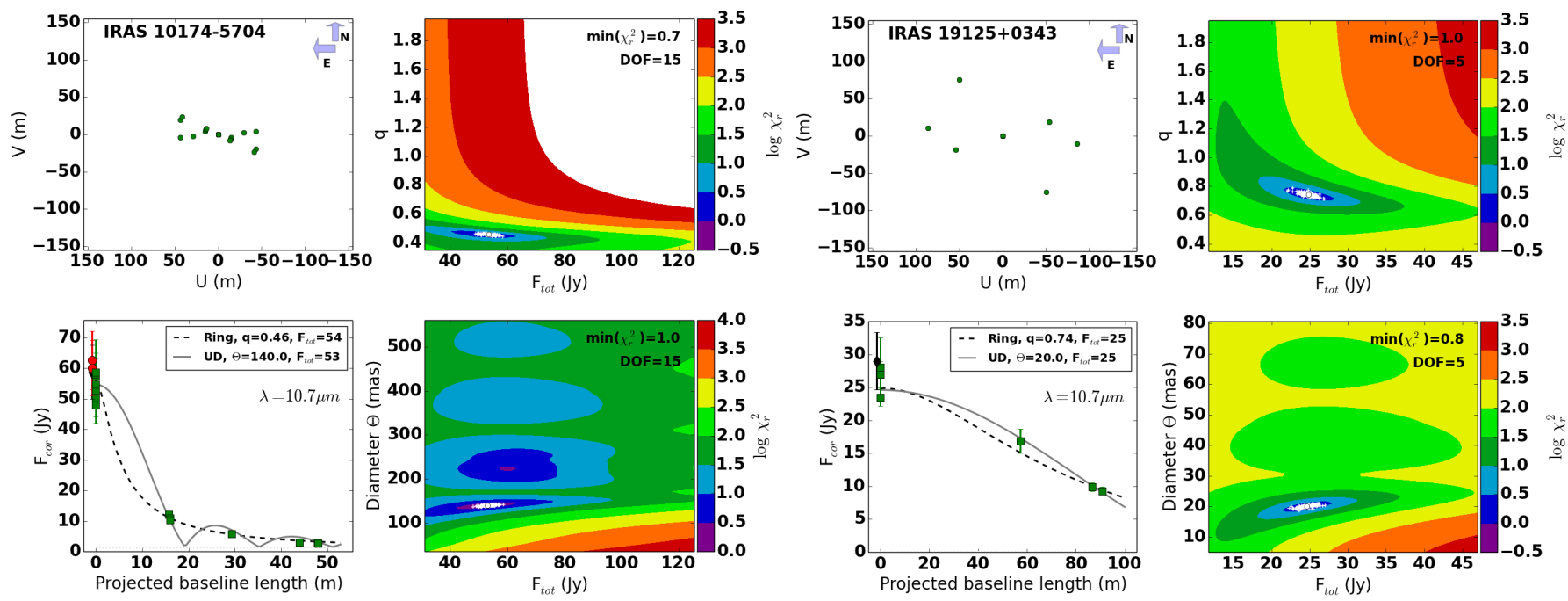

Fig. 6. Results of the interferometric fit for two sources, IRAS 10174-5704 (left) and IRAS 19125+0343 (right). The former is an outlier in our sample, while the latter is a typical and representative source. For each figure, the left panels show the 2D uv-coverage (upper) and the corresponding correlated fluxes as a function of projected baseline length (lower), respectively. The right panels show $\chi^{2}$-maps for the two models used in this paper, a uniform disk (upper) and the semi-physical disk model (lower). The minimum reduced $\chi^{2}$ and the degrees-of-freedom are indicated in the upper right corner of both panels. The white crosses in both $\chi^{2}$-maps are 100 random realisations of the posterior probability distribution that was sampled by the MCMC chain. The final adopted values are indicated with a white dot. Visibility curves corresponding to these final parameter values are included in the lower left panel as the full grey and black dashed lines for the uniform disk and semi-physical disk model, respectively.

with a period of $1250 \pm 300$ days (Kiss et al. 2007) which is likely the orbital period (Gezer et al. 2015). The angular diameter of the post-AGB photosphere, obtained via the SED modelling, is therefore not very well constrained. Our angle coverage in the UV plane is too small to detect deviations from spherical symmetry. AR Pup is very well resolved despite the short baselines used (Fig. B.3).

\subsection{HR4049 (nr. 7)}

The infrared properties of this source are well described in the literature (e.g. Dominik et al. 2003; Menut et al. 2009; Malek \& Cami 2014). Apart from a compact disk with a very small temperature range (Dominik et al. 2003), a large-scale outflow has been resolved in this source by spatially extended emission of the mid-IR Polycyclic Aromatic Hydrocarbon (PAH) features (e.g. Acke et al. 2013).

A similar over-resolved flux component is detected with MIDI, since the fitted zero-baseline flux of this source is significantly below the actual total-flux measurements (Fig. B.3). This is significant as the correlated fluxes of this object have a very high $\mathrm{S} / \mathrm{N}$. The fitted zero-baseline flux can be considered a good proxy for the total flux of the marginally resolved disk in this object.

\subsection{IRAS10174-5704 (nr. 8)}

The infrared spectrum of this source stands out in our sample. The amorphous silicates dominate with no evidence for crystalline processing nor for the presence of large grains. Gielen et al. (2011) suggest that this source might not be a postAGB star but a massive luminous supergiant in the process of losing mass. Also in our MIDI modelling, this object stands out (see Figs. 6 and B.3). It is very well resolved and the correlated fluxes reach to the third lobe of the best ring model. This corroborates the suggestion of Gielen et al. (2011) that this object is likely an outflow source with an optically thin wind.

\section{5. $89 \mathrm{Her}(\mathrm{nr} .16)$}

This object was already subject to a very extensive multiwavelength interferometric study (Hillen et al. 2013, 2014). One of the main conclusions was that in the optical, a resolved component is present which accounts for $35-40 \%$ of the visible photons. This component is associated with optical scattering by the circumbinary disk and an outflow or jet emanating from the central system. For 89 Her, we present two fits (see Fig. B.3): one with and one without taking the scattered light into account, taking the $T_{\text {eff }}$ and angular diameter from here or as determined in (Hillen et al. 2013). The $\chi^{2}$ improves when the scattered light is taken into account and the resulting $q$ value decreases to a less extreme value.

\section{6. $A C \operatorname{Her}(n r .17)$}

The results on ACHer are depicted in Fig. B.3. The source is very well resolved and the ring model allows to account for the higher correlated flux point of the $60 \mathrm{~m}$ baseline coming from the second lobe in the visibility profile. This source has been extensively modelled by Hillen et al. (2015). The radiative transfer modelling combined with the interferometric observables allowed to conclude that the disk in the AC Her system is in a very evolved state, as shown by its small gas/dust ratio and large inner hole. Indeed the inner rim is at significantly longer distance from the central source then the sublimation radius.

In our parametric modelling of the sample, which assumes that the dust disk starts at the sublimation radius, these physical properties of the disk around AC Her translate in a low $q$ value (so flat temperature gradient) and a large half-light radius. The fitted uniform disk radius is significantly smaller than the halflight radius. 


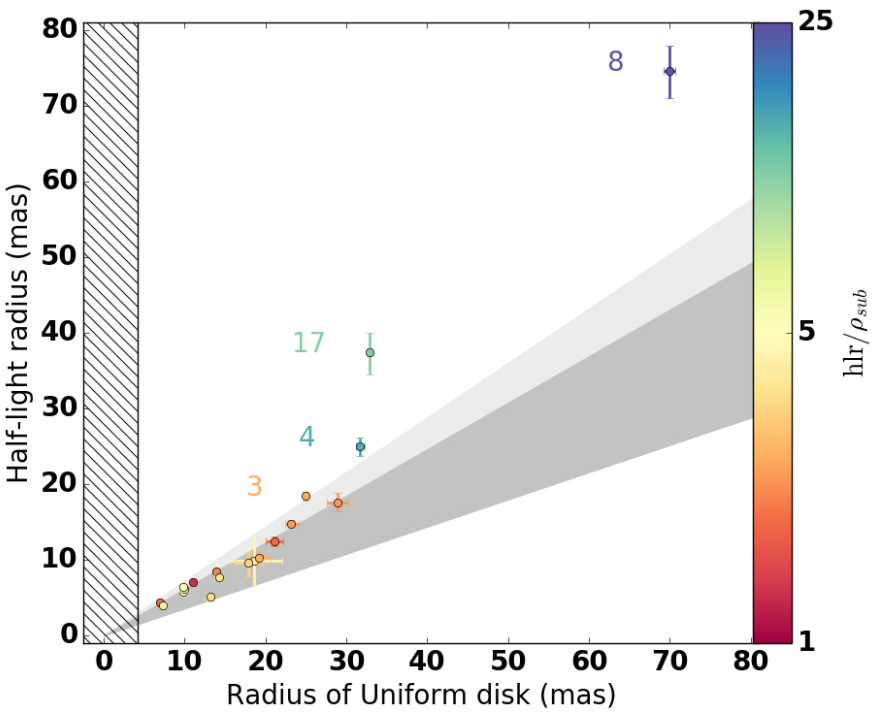

Fig. 7. Radius of the fitted uniform disk versus the half-light radius. The different regions and colours are described in the text (Sect. 7.1).

\section{Sample analysis}

\subsection{The half-light radius}

In Fig. 7 we compare the radius of the uniform disk with the halflight radius. The dark grey area denotes the region theoretically occupied by ring models for which the visibility $V>0.4$. It is computed by fitting the uniform disk model to an ensemble of ring models. The grid of ring models covers the full range of $\rho_{\text {sub }}$ of our sample and with $q \in\{0.4 ; 2.5\}$. The light grey area is an extension of this grid up to $V>0.3$. The hatched area denotes the region inaccessible by the interferometer. The colour of the observed sources (see colour bar on the right of Fig. 7) denotes the ratio of the half-light radius over the sublimation radius (see the colour bar on the right).

Most objects occupy the dark grey region and are thus only moderately resolved. Their half-light radii are a bit larger than the sublimation radius (typically a factor of $\sim 3$ ). This is expected for a disk with a fairly optically thick inner rim located at the sublimation radius and an outer disk surface that is not flaring very strongly. The region near the inner rim of the disk dominates the flux at $10.7 \mu \mathrm{m}$.

Four sources which stand out are U Mon (nr. 3), AR Pup (nr. 4), IRAS 10174-5704 (nr. 8), and AC Her (nr. 17) (see previous section for the details). These are the sources with the smallest $q$ values (see Table 4) and the largest ratio of half-light radius over sublimation radius. Since these objects have the largest appearance on the sky, they are also the most resolved - hence have visibilities below 0.4 - so that the details of the source morphology become important. As discussed previously, for two of these sources, IRAS 10174-5704 and AC Her, the $10.7 \mu$ m flux seems to emanate predominantly from radii well beyond the sublimation radius. The other two objects most likely only deviate because they are nearby, thus strongly resolved, and well inclined.

\subsection{Brightness temperatures}

We can use the uniform disk diameter to quantify the brightness temperature using the total flux at $10.7 \mu \mathrm{m}$ (see Fig. 8). The numbers in the figures indicate the index of the source in Table 4 and are plotted to the left and right of the corresponding location for even and uneven indexes, respectively. The sources are

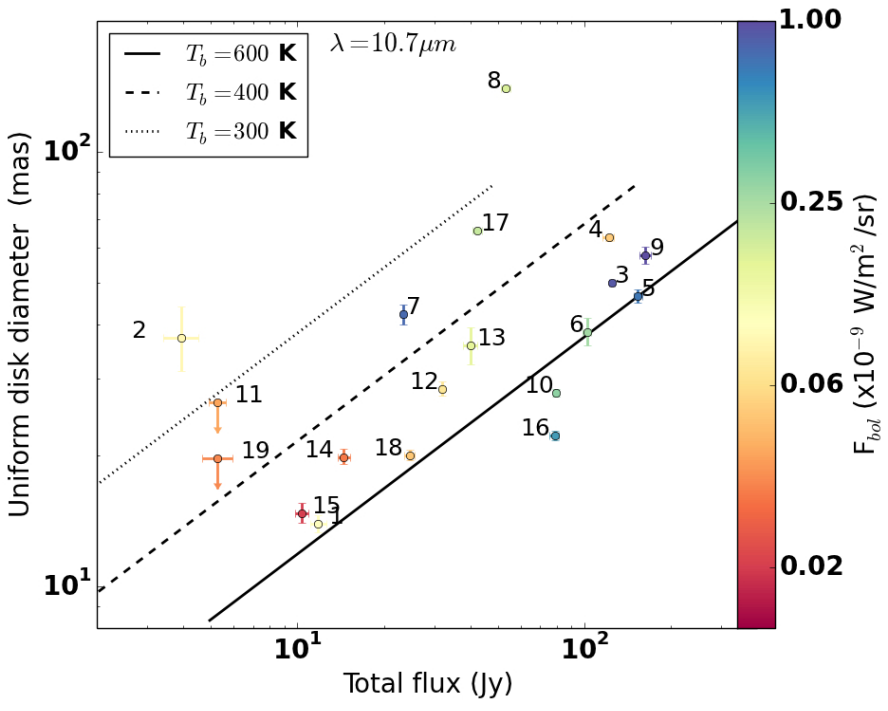

Fig. 8. Uniform disk diameter versus total flux at $10.7 \mu \mathrm{m}$. The numbers indicate the index of the source in Table 4. Symbols are explained in the text (Sect. 7.2).

coloured according to the stellar bolometric flux (see the colour bar on the right). The black dotted, dashed, and full lines represent uniform disks with brightness temperatures of 300, 400 and $600 \mathrm{~K}$, respectively. The dynamic range in $10.7 \mu \mathrm{m}$ flux is similar to that in bolometric flux $(\sim \times 50)$.

Overall the sources show a limited range in brightness temperature ( $\geq 50 \%$ of the sample falls between 400 and $600 \mathrm{~K})$ and an increase in flux is mainly due to an increase in the extent of the source. The correlation between the $10.7 \mu \mathrm{m}$ and stellar bolometric flux indicates that the observed range in angular extent mainly reflects variations in distance and stellar luminosity of the source, and that the disks are physically very similar within the sample.

Noticeable outlier is again IRAS 10174-5704 (nr. 8), but also HD 52961 (nr. 2) which is rather large for its flux. We suspect this is due to the contribution of PAH emission (Gielen et al. 2009) which likely has a different spatial scale than the thermal emission of the dust disk, similarly that HR 4049 (nr. 7, Sect. 6.3).

\subsection{A size-colour relation}

In Fig. 9 we compare the half-light radius to the $8-13 \mu \mathrm{m}$ colour $\left(-2.5 \log \left(F_{v, 8} / F_{v}, 13\right)\right)$. The horizontal axis represents the spectral slope in the $N$-band. To physically compare sources on the sizecolour diagram, the effect of the highly uncertain distance to the source should be removed. Hence, the distance is divided away by normalizing the physical half-light radius with the square root of the luminosity. Arrows indicate $3 \sigma$ upper limits. The numbers correspond to the index of the source in Table 4 and are plotted to the left and right of the corresponding source for even and uneven indexes, respectively. The colours indicate the $q$-value in the interferometric fit.

The homogeneity of the sample is again striking: all objects cluster in the same region, with very similar temperature power indices of the ring model. The largest sources (IRAS 10174-5704 (nr. 8) and AC Her (nr. 17) are also the reddest. AR Pup (nr. 4), on the other hand, appears large but has a similar IR-colour as the others. As this source is seen close to edge-on, the luminosity determined by integrating under the dereddened photosphere 


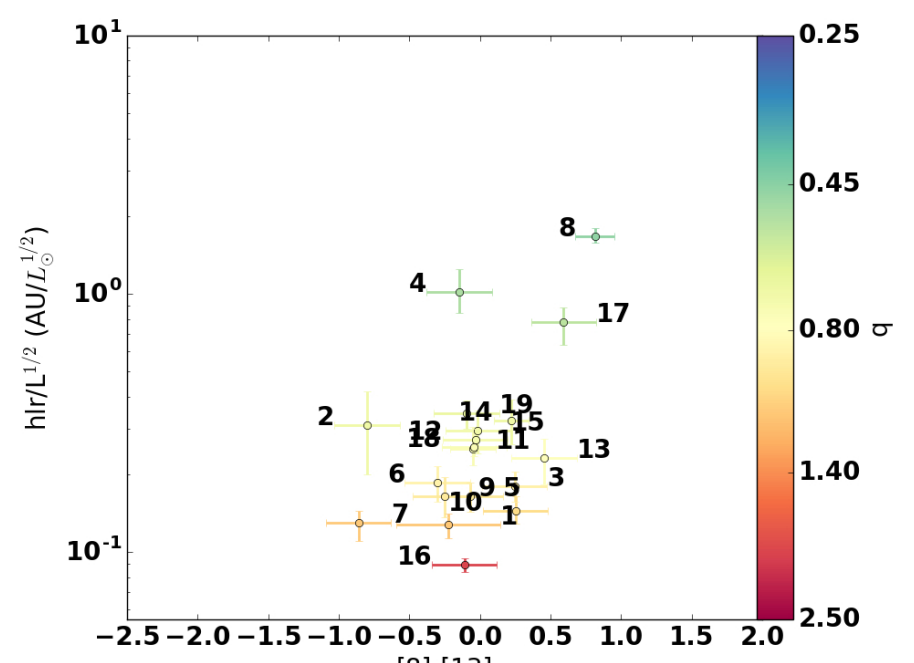

[8]-[13]

Fig. 9. Size-colour diagram of our sample of post-AGB stars. We plot the half-light radius $(h l r)$, normalized with the square root of the luminosity, versus the $8-13 \mu$ m colour $\left(-2.5 \log \left(F_{v, 8} / F_{v}, 13\right)\right)$. See Sect. 7.3.

(see Sect. 4) is a strong underestimate of the total luminosity of the central object.

\section{Comparison with a RT model grid}

The most important conclusion from our global analysis is that the sample of objects is very homogeneous, both in SED characteristics and in $N$-band interferometric behaviour. Our previous detailed radiative transfer modelling (Hillen et al. 2014, 2015) of 89 Her and AC Her showed that a model of a passively irradiated disk in hydrostatic equilibrium can provide a good match with both the energetics and the multi-colour interferometric observables of both objects.

Given the limited spatial constraints in our MIDI survey, we will not provide detailed models for all objects here, but we do want to test our assertion that the model of a passively irradiated disk gives a good match for the whole sample.

We therefore compute a grid of physical models. Similarly to our previous work, we used the radiative transfer code MCMax (Min et al. 2009) to compute disk structures. MCMax is based on the Monte Carlo method (Lucy 1999; Bjorkman \& Wood 2001). Photon packages are randomly emitted by a source at the origin of the coordinate system, which are then absorbed or scattered by dust that is distributed in an axi-symmetric geometry. The thermal structure is hence determined from the interaction of the dust with the stellar radiation (i.e. a passively heated disk in which the gas is in thermal equilibrium with the dust). The radial distribution of the gas and dust is a basic input of the model, but the vertical structure is obtained by solving the equation of hydrostatic equilibrium (i.e. the vertical component of the local gravitational force is balanced by the local gas pressure gradient). The temperature and density profiles in the disk are iteratively determined; taking a grain size distribution with size-dependent settling into account in a self-consistent way (Mulders \& Dominik 2012).

Overall the same strategy is adopted as in (Hillen et al. 2015) and we summarize the main properties of our radiative transfer models:

- the disk is vertically in hydrostatic equilibrium;

- we assume isotropic photon scattering by the dust;

- the composition of the dust is assumed to be an ISM-like mixture of silicates in the DHS (Distribution of Hollow
Spheres) approximation (Min et al. 2007) (the alternative mixture has the same composition but with an additional $15 \%$ of amorphous Carbon);

- the size of the dust grains follow a power-law distribution of which the exponent is varied;

- a grain-size-dependent settling of dust, counteracted by turbulence (with a variable turbulence strength parameter), is included self-consistently (Mulders \& Dominik 2012);

- we adopt a double-power-law formalism to parametrize the surface density distribution.

\subsection{The RT grid}

In Table 5 we list the grid parameters and their assumed values. The grid of models contains both geometric dimensions, in the form of a range of inclinations and baselines (angles and lengths) and a series of physical dimensions, related to the structure and composition of the disk model.

The dominant contributor to the $N$-band flux comes from the warm dust near the inner rim. Our model grid therefore focuses on the structure of the inner part of the disk, and on the dust properties. Hence we fix the outer radius as well as the outer surface density power law. We do apply different values for the inner surface density power law and for the turnover radius. Further dimensions in our physical grid comprise the grainsize distribution, the turbulence strength parameter $\alpha_{\text {turb }}$ and the dust mass. We assume that the disk inner radius is at the sublimation radius, according to Eq. (5.1).

We fix the central star properties, i.e., the central mass (total gravitational mass of the binary: $0.6+0.6 M_{\odot}$ ), the luminosity, and the effective temperature of the post-AGB component. The assumed temperature is equal to the median over the observed sample. The luminosity is taken to be a typical post-AGB value of $6000 L_{\odot}$. The distance is then fixed by matching the median angular diameter in our sample with the assumed luminosity. We compute the infrared [8]-[13]-colour for all our models. We fit the ring model to each synthetic visibility. Despite assuming values for certain parameters, we still have a total of 4320 models per dust composition type (times 98 baselines).

\subsection{Model comparison}

We now compare the diversity in the models to the sample of observed post-AGB stars. To do so, we fit the ring model to each synthetic visibility.

The left panel of Fig. 10 shows the position of each synthetic measurement in the size-luminosity diagram, again colour coded according the $q$ value. The grey region embraces the range covered by the model grid. The figure only shows the models computed with the first dust mixture (Table 5). The models computed with the second dust mixture shift the overall coverage only slightly upwards, so we limit our analysis to the models given in the figure.

Models that give rise to an apparently small disk (i.e., a high $q$ value and steep temperature gradient) are blue and vice versa.

In the right panel of Fig. 10 we illustrate that the grid of passive disks in hydrostatic equilibrium cover very well our stars in this representation of the interferometric observables.

A full parameter study is beyond the scope of this paper, but we shortly discuss some general characteristics of the model grid. Although our model disks are all continous, and start at the sublimation radius, they do cover a significant range in size, colour and temperature slope ( $q$-value). To illustrate the 

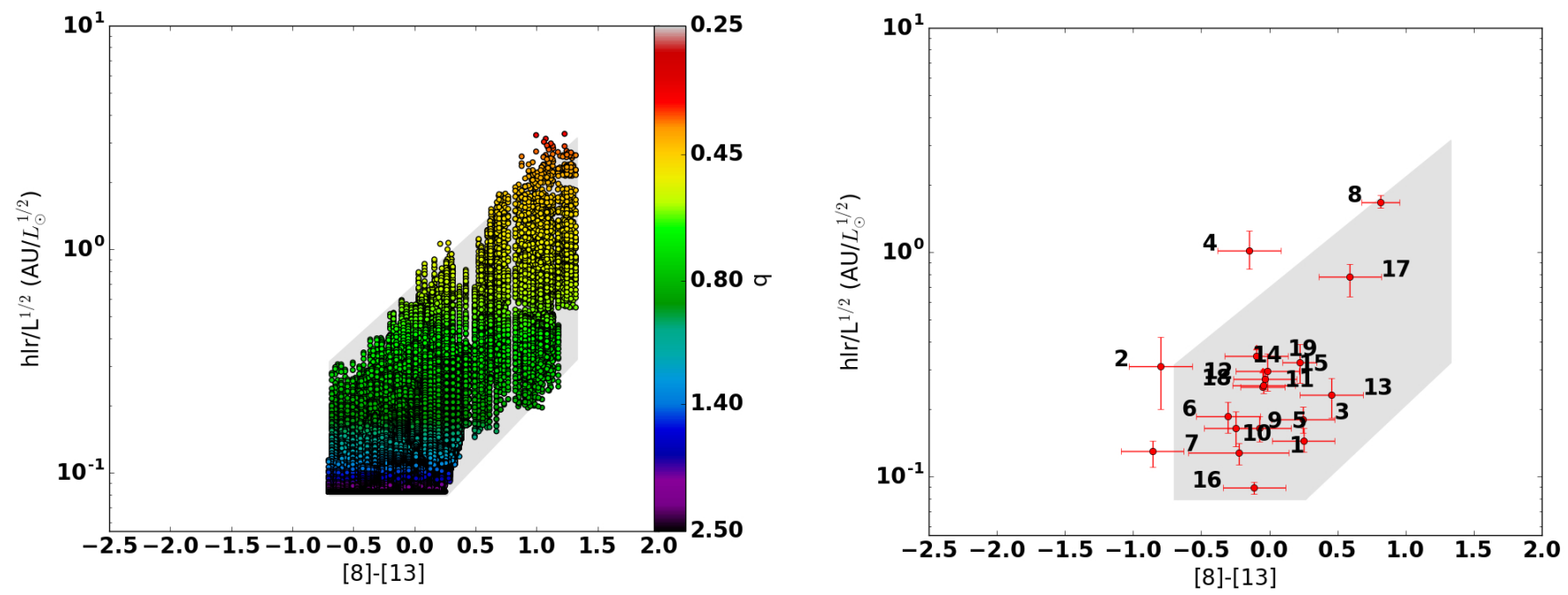

Fig. 10. Left: size-colour diagram with the distribution of observables computed for all models in the radiative transfer grid (i.e., one point corresponds to one combination of MCMax disk parameters and one baseline length+orientation). The colour coding is according to the $q$-value of the fit. Right: the location of the post-AGB disk stars within the range of models, shown as grey-shaded region.

Table 5. Input parameters of the radiative transfer model grid.

\begin{tabular}{ll}
\hline \hline Parameter & Value \\
\hline Star & \\
Mass $M_{\star}$ & $1.2 M_{\odot}$ \\
Luminosity $L$ & $6000 L_{\odot}$ \\
Effective temperature $T_{\text {eff }}$ & $6250 \mathrm{~K}$ \\
Distance $d$ & $2 \mathrm{kpc}$ \\
\hline Disk & \\
Outer surface density power $p_{\text {out }}$ & 1.0 \\
Inner surface density power $p_{\text {in }}$ & $1.0,0.0,-1.0,-2.0$ \\
Dust mass $M_{\text {dust }}$ & $10^{-4}, 10^{-3} M_{\odot}$ \\
Inner radius $R_{\text {in }}$ & $R_{\text {sub }}$ \\
Outer radius $R_{\text {out }}$ & $300 \mathrm{AU}$ \\
Turnover radius $R_{\text {mid }} / R_{\text {in }}$ & $2.0,2.5,3.0$ \\
Minimal grain size $a_{\text {min }}$ & $0.01,0.10,1.00 \mu \mathrm{m}$ \\
Maximal grain size $a_{\text {max }}$ & $1 \mathrm{~mm}$ \\
Grain size distribution $q_{\mathrm{g}}$ & $-3.50,-3.25,-3.00,-2.75$ \\
Dust-to-gas ratio & 0.01 \\
Turbulence parameter $\alpha$ & $10^{-4}, 10^{-3}, 10^{-2}$ \\
Inclination & $10^{\circ}, 30^{\circ}, 45^{\circ}, 55^{\circ}, 65^{\circ}, 75^{\circ}$ \\
Dust composition & $(1) \mathrm{Min}$ et al. $(2007)$ \\
& $(2) \mathrm{Min}$ et al. $(2007)+$ \\
& $15 \%$ amorphous Carbon \\
\hline Interferometric model observations & \\
Baseline lengths & $10,20,30, \ldots, 130,140 \mathrm{~m}$ \\
Baseline angles & $0,15,30,45,60,75,90^{\circ}$ \\
\hline
\end{tabular}

dependence of the fitted $q$ value on the RT model parameters, we show the distribution of $q$ as function of dust mass in Fig. 11. Only the more massive models (our gas-to-dust ratio is constant) lead to very low $q$ values (hence large disk appearances). Additionally, low $q$ values only occur for highly-inclined disks, with baselines oriented along the major axis of the disk. The peak at the higher end of $q$ is artificial and represents the tail of the distribution, as we assume $q<2.5$ in our model fitting. Such $q$ values predominantly come from highly-inclined, low-mass, single-power-law models with a small value for the $\alpha$ parameter, and with baselines oriented along the minor axis of the disk.

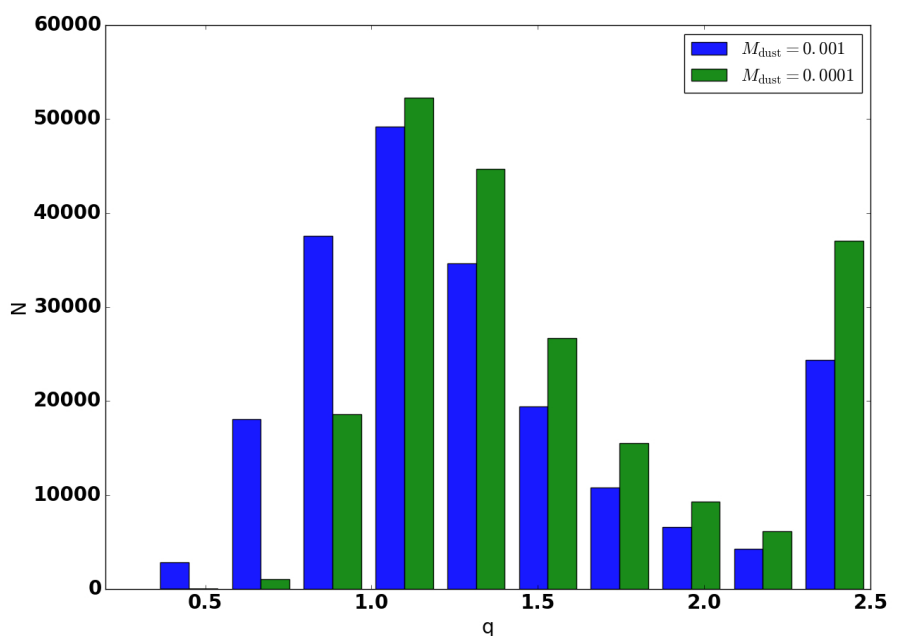

Fig. 11. Distribution of $q$-values obtained by our models. The colours differentiate the models with different dust mass.

Hence, models with an inner rim that approach a vertical wall in appearance.

We conclude that our model comparison shows that the physical structures computed by assuming a passively irradiated disk give a very good representation of post-AGB disks in terms of $N$-band interferometric observables and SED. This corroborates that the processes determining the physical structure of these disks are very similar to those operating in young YSOs, and that the models developed to represent proto-planetary disks around YSOs can be used very well for constraining the structure of disks at the opposite end of stellar evolution.

\subsection{Comparison with YSOs}

The main difference between YSOs of the Herbig Ae class analysed in the MIDI survey of Menu et al. (2015) and the post-AGB stars analysed here, is that the latter display significantly larger central luminosities, while the central gravitational mass is not too different. This means that the inner rims of post-AGB disks, which are at the sublimation radius, lie further away from the 


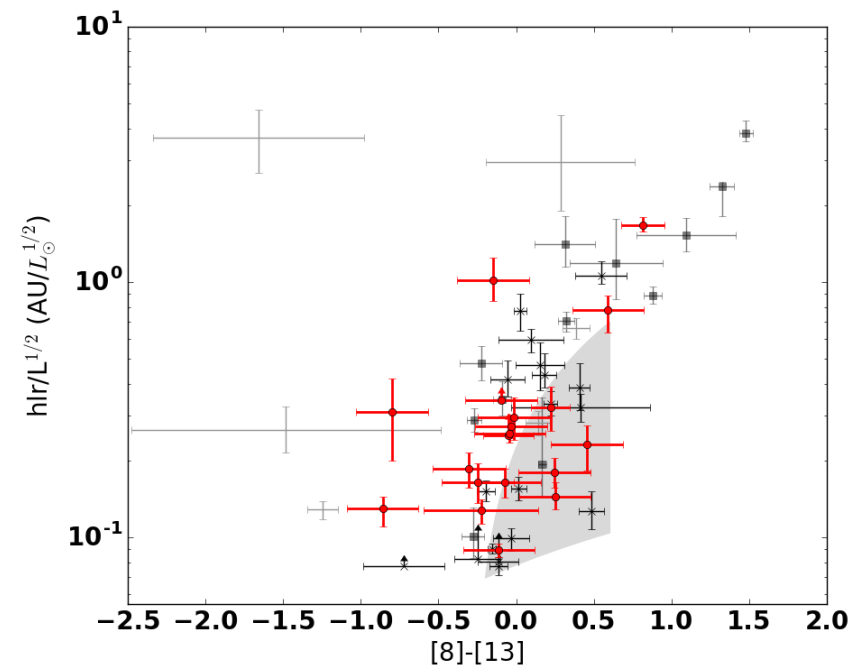

Fig. 12. Size-colour diagram of our sample of post-AGB stars compared to the sample of Herbig Ae disks that was studied by Menu et al. (2015). Arrows indicate upper limits. The symbols are explained in Sect. 8.3. The grey area delimits the region of continuous HAEBE disk models computed by Menu et al. (2015).

illuminating star, where the local gravity field is smaller than for YSOs. The hot inner rim has a significant scale-height. This is also evident from the large infrared excess, compared to the energy that is available from the photosphere.

A direct comparison of the disks around YSO and postAGB stars in the size-colour diagram is given in Fig. 12. We used the sample of Herbig Ae stars of Menu et al. (2015). A differentiation is made between three groups of Herbig Ae sources (the classification of Meeus et al. 2001): group Ia (black squares), Ib (grey plusses) and II (black crosses). This classification of YSOs was made on the basis of the far-IR SED (Meeus et al. 2001), and has been interpreted in terms of flaring (group I) vs. self-shadowed (group II) disks (Dullemond et al. 2001; Dullemond \& Dominik 2004a,b). More recent studies of transition and pre-transition disks suggest that these group I SEDs show a large gap in the disk and display an inner disk and a large outer disk (e.g. Kraus \& Ireland 2012; Menu et al. 2015; van der Marel et al. 2016, and references therein).

Obviously, the $10 \mu \mathrm{m}$ size-colour diagram is insufficient to demonstrate the full differences between the variety of YSO and post-AGB disks, but it is striking that these objects cluster very similarly, despite the different evolutionary stage of the host star(s). More precisely, and similar to their SED behaviour (de Ruyter et al. 2006), the post-AGB sources cover very much the same region as the group II Herbig Ae stars (with a few outliers that were discussed in Sects. 6 and 7). It thus seems that post-AGB disks are significantly puffed-up at their inner rim, but do not have a strongly flaring outer surface or evidence for a large outer disk.

The dark grey zone in Fig. 12 represents the region of continuous disks as computed in Menu et al. (2015). They used the same code for the disk modelling, but with distinct assumptions. The main differences are the central star properties, our choice for a double-power-law formalism, the model parameter coverage (e.g. the dust mass) and the assumed presence of a halo at the sublimation radius. The combination of these different choices explains why our continuous models cover a larger zone in the size-colour diagram. Additionally, Menu et al. (2015) computed a range of models with a gap (i.e. the inner radius of the disk

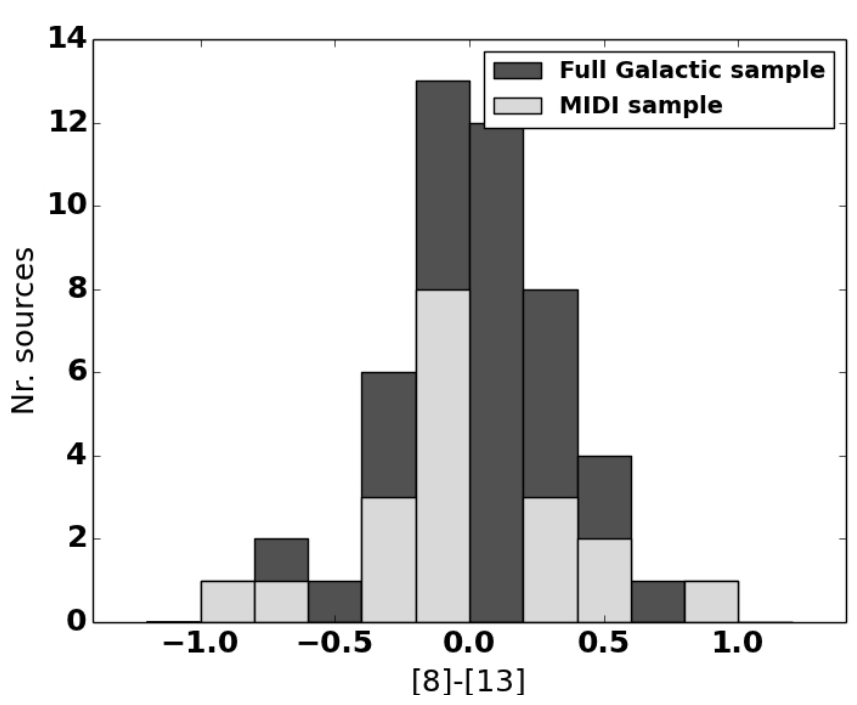

Fig. 13. Histogram of the [8]-[13] colour, comparing our MIDI sample to the known Galactic population of which spectra were presented by Gielen et al. (2011).

lies beyond the sublimation radius), to explain the largest and reddest objects. Our analysis shows, however, that being in the upper right corner of the size-colour diagram is not a sufficient condition to conclude about the presence of a gap in the disk. Sufficiently massive and highly inclined disks (see Fig. 11), observed with baselines aligned along the major axis, also appear big and red in the size-colour diagram. On the other hand, there are several Herbig Ae sources and one post-AGB object (nr. 17 AC Her, Hillen et al. 2015), for which a more detailed analysis of the wavelength-dependent MIDI visibilities and differential phases have shown the presence of a gap. In general, however, a good uv-coverage (or an a priori constraint on the disk inclination and/or mass) is required to firmly establish the presence of gaps in individual sources. This will be possible with the 2nd-generation VLTI instrument MATISSE.

\section{Discussion}

We present a large interferometric $N$-band survey of 19 postAGB objects, which are classified as proven or suspected binary stars with a circumbinary disk. This sample is representative of the whole Galactic population of similar objects (Fig. 13). For the interpretation of the correlated fluxes, we also constructed the full SEDs in a homogeneous way. We differentiated in the $\mathrm{N}$-band between the faint and bright state in the pulsation cycle for two high-amplitude variables (U Mon (nr. 3) and IRAS 17038 (nr. 14)).

A large fraction (17/19) of the objects is resolved with our MIDI interferometric baselines, showing a very compact emission region, corroborating the compact-disk interpretation of the SED. Our model fitting and sample-wise comparison to a grid of continuous radiative transfer models shows that the inner rim in these disks is at the sublimation radius and dominates the $\mathrm{N}$-band for most of the sources. Although diverse in $\mathrm{N}$-band total flux, the objects show a very homogeneous behaviour in the interferometric observables. This shows that the disk structures are very similar within the sample. Noticeable exceptions are IRAS 10174 (nr. 8) and objects for which PAH emission, with a proven or suspected different spatial distribution, affects the result (HR4049 (nr. 2) and HD 52961 (nr. 4)). There is also one 
rather evolved disk in our sample, AC Her (nr. 17, Hillen et al. 2015), which has an inner rim that is significantly larger than the sublimation radius. The homogeneity in the sample is striking, given the covered range in photospheric temperature and orbital period. Although we do not constrain the post-AGB age, the sample homogeneity also indicates that the structure of these disks is very stable.

\subsection{Aspect angle}

The inclination angle of the disk will of course impact on the observational properties. Unfortunately, our angular coverage of the uv-plane is too limited to determine the inclinations systematically. For objects like AR Pup (nr. 4) an edge-on viewing angle can be anticipated, but it is striking that actually many of the systems in Table 1 (10/19) show long-term photometric behaviour that can be linked to orbital motion. A natural explanation of this so-called "RVb phenomenon" is that a grazing aspect angle over the disk will affect the line-of-sight extinction during the orbital motion. This will then result in light curves in which the orbital period is visible and in objects that appear redder when fainter. A complicating factor is, however, that the optical scattering, which can represent up to $35-40 \%$ of the optical flux (Hillen et al. 2014), is known to be strongly angle-dependent. The large fraction of RVb objects must mean that orbital effects can show up in the lightcurves for a wide range of inclinations.

Our RT model grid of passively irradiated disks in hydrostatic equilibrium covers very well the observed properties. The bulk of the objects lie in the region of the more compact models, in which the inner rim dominates the $N$-band flux (Fig. 10). We note, however, that the most compact models (those with $q \sim 2.5$ ) are not compatible with the observations, which indicates that there is some smoothing of the inner rim (i.e. the rim does not show up as a vertical wall).

\subsection{Comparison with YSO}

To quantify the similarity between our evolved systems and YSOs, we compared them directly in the distance-independent $\mathrm{N}$-band size-colour diagram in Fig. 12. Both in their integrated SED properties and in the $N$-band intensity distribution on the sky, the post-AGB stars resemble very well the settled group II disks around Herbig Ae stars. Disks of group Ia/Ib with a large far infrared excess, are not observed around post-AGB stars. If the group Ia/Ib YSO disks can be linked to active planet formation, as suggested in Menu et al. (e.g. 2015), this means that planet formation does not occur in the same way around postAGB stars, if it happens at all. Indeed, the fast evolution of the central evolved stars may prevent the formation of grains beyond the $\mathrm{mm}$ - and cm-sized particles that are commonly detected (whenever sub-mm fluxes are available, see e.g. Hillen et al. 2015, 2014; Sahai et al. 2011).

\subsection{Binary connection}

Second-generation protoplanetary disks are only found around post-AGB stars that are part of a binary system with an orbital period roughly in-between a hundred and a few thousand days (van Winckel 2003; van Winckel et al. 2009). Such orbits are too small to accommodate an AGB star. All these objects must have gone through a phase of strong binary interaction. No spiralled-in system nor merged system has yet been detected among the objects with a disk-like SED that we are monitoring in a large radial velocity programme (Van Winckel et al. 2010).
Spiralled-in systems in PNe typically display periods of a few days only, hence they have large radial-velocity amplitudes and the lack of such systems among the known post-AGB binaries is not an observational bias. Even if the formation of these secondgeneration disks remains elusive, the disparity between these populations of binary systems suggests that the formation of a disk (and the angular momentum contained in it), may prevent an evolution towards dramatic spiral-in.

\subsection{Formation and evolution}

Although the focus of this paper is on the current structure of post-AGB circumbinary disks, the impact of these disks on the further evolution of their host stars should not be underestimated. Many post-AGB stars display a chemical anomaly in their photosphere (van Winckel 2003). The abundance trends in such atmospheres resemble the gas phase of the interstellar medium: refractory elements are underabundant, while volatiles retain their original abundances. Such depletion patterns are intimately linked to the presence of a disk around the post-AGB star, but the latter is not a sufficient condition to observe depletion (e.g. Gezer et al. 2015). The anomaly can last long, as is illustrated by its presence in $\mathrm{BD}+33^{\circ} 2642$, the central star of a PN. It is long known that the chemical abundances show depletion in this object (Napiwotzki et al. 1994), but it was only recently proven to be a spectroscopic binary with a period of $1105 \pm 24$ days (Van Winckel et al. 2014).

There is also increasing evidence that the presence of a circumbinary disk has a lasting effect on the dynamical evolution of the binary orbit, as resonant coupling between the binary and the disk can pump-up the eccentricity (e.g. Artymowicz et al. 1991; Lubow 2010; Dermine et al. 2013; Vos et al. 2015). This provides a way to explain the commonly observed large eccentricities (e.g. van Winckel et al. 2009; Gezer et al. 2015, and references therein for the orbits). The integrated effect depends very much on the mass and longevity of the disk, as well as on the radial mass distribution within the disk.

The MIDI spectral coverage and sensitivity makes that only objects with significant $N$-band excesses can be resolved. But disks also evolve, as is likely illustrated by the $\mathrm{BD}+39^{\circ} 4926$ system. This is a highly depleted post-AGB binary in an orbit of $\sim 870$ days for which only recently some circumstellar material was detected via a small excess at $22 \mu \mathrm{m}$ (Kodaira et al. 1970; Venn et al. 2014; Gezer et al. 2015). The lack of an excess at shorter wavelengths, for a depleted star, indicates the presence of a highly evolved disk in which the innermost material has been removed. The remaining material is probably present in some form of debris disk.

It is by now well established that there are also white dwarfs (WD) with circumstellar material. The first discovery was made through the detection of an IR-excess (Zuckerman \& Becklin 1987), and evaluated to be a disk created by the destruction of asteroids or even planets (Graham et al. 1990). These debris disks are now found around more than 30 WD (e.g. Manser et al. 2016, and references therein), but they are typically very close to the WD. When the WD accretes some of the solid material, the opposite happens to what is detected in the post-AGB binaries discussed in this paper: the refractory elements become more abundant. This is in fact an observable to trace circumstellar material around WD (e.g. Clayton et al. 2014). The tidal disruption can even be detected as multiple transits caused by clouds of debris material (see the lightcurve of WD1145+017, Gänsicke et al. 2016). There is no link to binary evolution for these systems. 
This is different for post-common envelope WD plus M-dwarf systems. Interesting here is that the transit timing variations of NN Ser can be explained very well with a two-planet model (Marsh et al. 2014). The recent detection of circumbinary dust (Hardy et al. 2016) leads to the conclusion that these planets may well be of secondary origin and are formed from the material ejected in the common envelope event. This system has spiralled-in, so there is no evolutionary connection between post-AGB wide binaries, which avoided spiral-in, and NN Ser. However, the potential formation of secondary planets is also in the context of long-lived disks around post-AGB binaries an important item for focussed future research.

The dust in old WD discussed above, is located near the disruption radius, so close to the WD itself. Larger disks have been discovered as well, and mainly around WDs which are in the centre of a planetary nebula (e.g. Bilíková et al. 2012, and references therein). The disk in the Helix nebula is likely the most famous example (Su et al. 2007). The systematic study by Clayton et al. (2014) shows that the observational evidences are not conclusive, but these wider disks are linked to a formation scenario of secondary disks on the AGB (and not via destruction of asteroids) as well as to binary evolution (as most CSPNe with larger disks are binaries). The orbital properties of these CSPNe are, however, diverse and not complete.

\subsection{Conclusion}

We conclude that our $N$-band interferometric survey confirms the presence of long-lived disks around post-AGB stars. These post-AGB disks have an inner structure that resembles very well that of proto-planetary disks around YSO. As the luminous central star is in a fast evolutionary phase, these objects offer an alternative environment to study proto-planetary disk structure, evolution and evaporation. Moreover, with multi-beam combiners, these objects provide an ideal opportunity to obtain, via interferometric imaging, a direct probe for disk-binary interactions and their possible dependence of orbital phase (Hillen et al. 2016). Given the results of our interferometric and radial velocity surveys, we show that the radiative and dynamical interaction between these binary systems and their dusty environments can now be resolved for a significant fraction of the known Galactic population of these objects. These scaled-up versions of protoplanetary disks can be used to study poorly understood disk evolution and dispersal processes under a much wider range of physical conditions. The disks must have an important role in the future evolution of these stars and the potential evolutionary connection to the circumstellar material around WD needs to be further explored.

Acknowledgements. M.H., R.M., D.K. and H.V.W. acknowledge support from the Research Council of the KU Leuven under grant number GOA/2013/012. D.K. acknowledges support of the Research Foundation - Flanders under contract G.0B86.13. The authors acknowledge Christoffel Waelkens, Bram Acke, Pieter Deroo, Cees Dullemond and Tom Lloyd Evans (RIP) for enlightening discussions during the whole project. We acknowledge with thanks the variable sta observations from the AAVSO International Database contributed by observer worldwide and used in this research. This research has made use of the SIMBAD database, operated at CDS, Strasbourg, France. This research has made use of NASA's Astrophysics Data System Bibliographic Services. This research has made use of the Jean-Marie Mariotti Center Aspro service ${ }^{1}$.

\section{References}

Acke, B., Degroote, P., Lombaert, R., et al. 2013, A\&A, 551, A76 Armitage, P. J. 2010, Astrophysics of Planet Formation (Cambridge University Press)

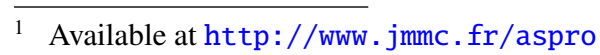

Artymowicz, P., Clarke, C. J., Lubow, S. H., \& Pringle, J. E. 1991, ApJ, 370, L35

Bakker, E. J., Lamers, H. J. G. L. M., Waters, L. B. F. M., \& Waelkens, C. 1996, A\&A, 310, 861

Bilíková, J., Chu, Y.-H., Gruendl, R. A., Su, K. Y. L., \& De Marco, O. 2012, ApJS, 200, 3

Bjorkman, J. E., \& Wood, K. 2001, ApJ, 554, 615

Boley, P. A., Linz, H., van Boekel, R., et al. 2013, A\&A, 558, A24

Bujarrabal, V., Castro-Carrizo, A., Alcolea, J., \& Neri, R. 2005, A\&A, 441, 1031

Bujarrabal, V., Alcolea, J., Van Winckel, H., Santander-García, M., \& Castro-Carrizo, A. 2013a, A\&A, 557, A104

Bujarrabal, V., Castro-Carrizo, A., Alcolea, J., et al. 2013b, A\&A, 557, L11

Bujarrabal, V., Castro-Carrizo, A., Alcolea, J., \& Van Winckel, H. 2015, A\&A, 575, L7

Burtscher, L., Meisenheimer, K., Tristram, K. R. W., et al. 2013, A\&A, 558, A149

Clayton, G. C., De Marco, O., Nordhaus, J., et al. 2014, AJ, 147, 142

Cutri, R. M., Skrutskie, M. F., van Dyk, S., et al. 2003, VizieR Online Data Catalog: II/246

Cutri, R. M., et al. 2012, VizieR Online Data Catalog: II/311

de Ruyter, S., van Winckel, H., Dominik, C., Waters, L. B. F. M., \& Dejonghe, H. 2005, A\&A, 435, 161

de Ruyter, S., van Winckel, H., Maas, T., et al. 2006, A\&A, 448, 641

Degroote, P., Acke, B., Samadi, R., et al. 2011, A\&A, 536, A82

Dermine, T., Izzard, R. G., Jorissen, A., \& Van Winckel, H. 2013, A\&A, 551, A50

Deroo, P., van Winckel, H., Min, M., et al. 2006, A\&A, 450, 181

Deroo, P., Acke, B., Verhoelst, T., et al. 2007, A\&A, 474, L45

Díaz, F., Hearnshaw, J., Rosenzweig, P., et al. 2007, in IAU Symp. 240, eds. W. I. Hartkopf, P. Harmanec, \& E. F. Guinan, 127

Dominik, C., Dullemond, C. P., Cami, J., \& Van Winckel, H. 2003, A\&A, 397, 595

Drimmel, R., \& Spergel, D. N. 2001, ApJ, 556, 181

Dubath, P., Rimoldini, L., Süveges, M., et al. 2011, MNRAS, 414, 2602

Dullemond, C. P., \& Dominik, C. 2004a, A\&A, 417, 159

Dullemond, C. P., \& Dominik, C. 2004b, A\&A, 421, 1075

Dullemond, C. P., \& Monnier, J. D. 2010, ARA\&A, 48, 205

Dullemond, C. P., Dominik, C., \& Natta, A. 2001, ApJ, 560, 957

Evans, T. L. 1985, MNRAS, 217, 493

Fitzpatrick, E. L. 2004, in Astrophysics of Dust, eds. A. N. Witt, G. C. Clayton, \& B. T. Draine, ASP Conf. Ser., 309, 33

Foreman-Mackey, D., Hogg, D. W., Lang, D., \& Goodman, J. 2013, PASP, 125, 306

Fukugita, M., Ichikawa, T., Gunn, J. E., et al. 1996, AJ, 111, 1748

Gänsicke, B. T., Aungwerojwit, A., Marsh, T. R., et al. 2016, ApJ, 818, L7

Gehrz, R. D. 1971, Ph.D. Thesis, University of Minnesota, USA

Gezer, I., Van Winckel, H., Bozkurt, Z., et al. 2015, MNRAS, 453, 133

Gielen, C., van Winckel, H., Waters, L. B. F. M., Min, M., \& Dominik, C. 2007, A\&A, 475, 629

Gielen, C., van Winckel, H., Min, M., Waters, L. B. F. M., \& Lloyd Evans, T. 2008, A\&A, 490, 725

Gielen, C., van Winckel, H., Matsuura, M., et al. 2009, A\&A, 503, 843

Gielen, C., Bouwman, J., van Winckel, H., et al. 2011, A\&A, 533, A99

Golay, M. 1972, Vistas Astron., 14, 13

Goldsmith, M. J., Evans, A., Albinson, J. S., \& Bode, M. F. 1987, MNRAS, 227, 143

Graham, J. R., Matthews, K., Neugebauer, G., \& Soifer, B. T. 1990, ApJ, 357, 216

Haguenauer, P., Abuter, R., Andolfato, L., et al. 2012, in Optical and Infrared Interferometry III, Proc. SPIE, 8445, 84450D

Hardy, A., Schreiber, M. R., Parsons, S. G., et al. 2016, MNRAS, 459, 4518

Hillen, M., Verhoelst, T., Van Winckel, H., et al. 2013, A\&A, 559, A111

Hillen, M., Menu, J., Van Winckel, H., et al. 2014, A\&A, 568, A12

Hillen, M., de Vries, B. L., Menu, J., et al. 2015, A\&A, 578, A40

Hillen, M., Kluska, J., Le Bouquin, J.-B., et al. 2016, A\&A, 588, L1

Høg, E., Fabricius, C., Makarov, V. V., et al. 2000, A\&A, 355, L27

Jaffe, W. J. 2004, in SPIE Conf. Ser. 5491, ed. W. A. Traub, 715

Kamath, D., Wood, P. R., \& Van Winckel, H. 2014, MNRAS, 439, 2211

Kamath, D., Wood, P. R., \& Van Winckel, H. 2015, MNRAS, 454, 1468

Kamath, D., Wood, P. R., Van Winckel, H., \& Nie, J. D. 2016, A\&A, 586, L5

Kass, R. E., \& Raftery, A. E. 1995, J. Am. Stat. Assoc., 90, 773

Kenyon, S. J., \& Hartmann, L. 1987, ApJ, 323, 714

Kipper, T. 2005, Balt. Astron., 14, 223

Kiss, L. L., Derekas, A., Szabó, G. M., Bedding, T. R., \& Szabados, L. 2007, MNRAS, 375, 1338

Kodaira, K., Greenstein, J. L., \& Oke, J. B. 1970, ApJ, 159, 485

Kraus, A. L., \& Ireland, M. J. 2012, ApJ, 745, 5

Lagadec, E., Verhoelst, T., Mékarnia, D., et al. 2011, MNRAS, 417, 32 
Lagage, P. O., Pel, J. W., Authier, M., et al. 2004, The Messenger, 117, 12

Lamers, H. J. G. L. M., \& Cassinelli, J. P. 1999, Introduction to Stellar Winds (Cambridge University Press)

Leinert, C., Graser, U., Waters, L. B. F. M., et al. 2003, in Interferometry for Optical Astronomy II, ed. W. A. Traub, SPIE Conf. Ser., 4838, 893

Lubow, S. H. 2010, MNRAS, 406, 2777

Lucy, L. B. 1999, A\&A, 344, 282

Maas, T. 2003, Ph.D. Thesis, Institute of Astronomy, Katholieke Universiteit Leuven, Belgium

Maas, T., Van Winckel, H., Lloyd Evans, T., et al. 2003, A\&A, 405, 271

Malek, S. E., \& Cami, J. 2014, ApJ, 780, 41

Manick, R., Van Winckel, H., Kamath, D., et al. 2017, A\&A, 597, A129

Manser, C. J., Gänsicke, B. T., Marsh, T. R., et al. 2016, MNRAS, 455, 4467

Marsh, T. R., Parsons, S. G., Bours, M. C. P., et al. 2014, MNRAS, 437, 475

Meeus, G., Waters, L. B. F. M., Bouwman, J., et al. 2001, A\&A, 365, 476

Menu, J., van Boekel, R., Henning, T., et al. 2015, A\&A, 581, A107

Menut, J.-L., Chesneau, O., Bakker, E., et al. 2009, A\&A, 496, 133

Mermilliod, J.-C., Mermilliod, M., \& Hauck, B. 1997, A\&AS, 124, 349

Min, M., Waters, L. B. F. M., de Koter, A., et al. 2007, A\&A, 462, 667

Min, M., Dullemond, C. P., Dominik, C., de Koter, A., \& Hovenier, J. W. 2009, A\&A, 497, 155

Monnier, J. D., Tuthill, P. G., Ireland, M., et al. 2009, ApJ, 700, 491

Mulders, G. D., \& Dominik, C. 2012, A\&A, 539, A9

Murakami, H., Baba, H., Barthel, P., et al. 2007, PASJ, 59, 369

Nagel, E., D’Alessio, P., Calvet, N., et al. 2010, ApJ, 708, 38

Napiwotzki, R., Heber, U., \& Koeppen, J. 1994, A\&A, 292, 239

Neugebauer, G., Habing, H. J., van Duinen, R., et al. 1984, ApJ, 278, L1

Olofsson, H., Vlemmings, W. H. T., Maercker, M., et al. 2015, A\&A, 576, L15

Percy, J. R., \& Bakos, A. 1998, J. Am. Assoc. Var. Star Observers, 26, 112

Percy, J. R., Bakos, A. G., \& Henry, G. W. 2000, PASP, 112, 840

Pojmanski, G. 2003, Acta Astron., 53, 341

Pojmanski, G., \& Maciejewski, G. 2004, Acta Astron., 54, 153

Pojmanski, G., Pilecki, B., \& Szczygiel, D. 2005, Acta Astron., 55, 275

Pollard, K. R., Cottrell, P. L., Kilmartin, P. M., \& Gilmore, A. C. 1996, MNRAS 279, 949

Pollard, K. R., McSaveney, J. A., \& Cottrelll, P. L. 2006, Mem. Soc. Astron. It., 77, 527

Preston, G. W., Krzeminski, W., Smak, J., \& Williams, J. A. 1963, ApJ, 137, 401

Price, S. D. 1995, Space Sci. Rev., 74, 81

Sahai, R., Claussen, M. J., Schnee, S., Morris, M. R., \& Sánchez Contreras, C. 2011, ApJ, 739, L3

Samus, N. N., Durlevich, O. V., et al. 2009, VizieR Online Data Catalog: II/25
Schlegel, D. J., Finkbeiner, D. P., \& Davis, M. 1998, ApJ, 500, 525

Shenton, M., Albinson, J. S., Barrett, P., et al. 1992, A\&A, 262, 138 Shenton, M., Evans, A., Albinson, J. S., et al. 1994, A\&A, 292, 102 Smith, B. J., Price, S. D., \& Baker, R. I. 2004, ApJS, 154, 673

Strömgren, B. 1956, Vist. Astron., 2, 1336

Su, K. Y. L., Chu, Y.-H., Rieke, G. H., et al. 2007, ApJ, 657, L41

Taranova, O. G., Shenavrin, V. I., \& Tatarnikov, A. M. 2009, Astron. Lett., 35, 472

Taranova, O. G., Shenavrin, V. I., \& Tatarnikov, A. M. 2010, VizieR Online Data Catalog: J/PAZh/36/144

Thompson, G. I., Nandy, K., Jamar, C., et al. 1978, Catalogue of stellar ultraviolet fluxes, A compilation of absolute stellar fluxes measured by the Sky Survey Telescope (S2/68) aboard the ESRO satellite TD-1

van Aarle, E., van Winckel, H., Lloyd Evans, T., et al. 2011, A\&A, 530, A90

van der Marel, N., Verhaar, B. W., van Terwisga, S., et al. 2016, A\&A, 592, A126 van Winckel, H. 2003, ARA\&A, 41, 391

Van Winckel, H., Waelkens, C., Waters, L. B. F. M., et al. 1998, A\&A, 336, L17 Van Winckel, H., Waelkens, C., Fernie, J. D., \& Waters, L. B. F. M. 1999, A\&A, 343,202

van Winckel, H., Lloyd Evans, T., Briquet, M., et al. 2009, A\&A, 505, 1221

Van Winckel, H., Jorissen, A., Gorlova, N., et al. 2010, Mem. Soc. Astron. It., 81,1022

Van Winckel, H., Jorissen, A., Exter, K., et al. 2014, A\&A, 563, L10

Venn, K. A., Puzia, T. H., Divell, M., et al. 2014, ApJ, 791, 98

Vos, J., Østensen, R. H., Marchant, P., \& Van Winckel, H. 2015, A\&A, 579, A49

Waelkens, C., Waters, L. B. F. M., Cassatella, A., Le Bertre, T., \& Lamers, H. J. G. L. M. 1987, A\&A, 181, L5

Waelkens, C., Lamers, H. J. G. L. M., Waters, L. B. F. M., et al. 1991a, A\&A, 242,433

Waelkens, C., Van Winckel, H., Bogaert, E., \& Trams, N. R. 1991b, A\&A, 251, 495

Waelkens, C., Waters, L. B. F. M., van Winckel, H., \& Daems, K. 1995, Ap\&SS, 224,357

Wahlgren, G. M. 1992, AJ, 104, 1174

Waters, L. B. F. M., Waelkens, C., Mayor, M., \& Trams, N. R. 1993, A\&A, 269, 242

Wesselius, P. R., van Duinen, R. J., de Jonge, A. R. W., et al. 1982, A\&AS, 49, 427

Zsoldos, E. 1993, A\&A, 268, 149

Zsoldos, E. 1996, A\&AS, 119, 431

Zuckerman, B., \& Becklin, E. E. 1987, Nature, 330, 138 


\section{Appendix A: Origin of literature photometry}

In this appendix we give full reference to the sources of the photometry used in this work. Our Python-based SED fitting tools allow to automatically retrieve the available photometry in a predefined list of Simbad/Vizier catalogues. This is the case for the following photometry: (UV) from the TD1 (Thompson et al. 1978) and the ANS satellite (Wesselius et al. 1982), (optical and near-IR) from the JOHNSON/COUSINS 11band, GENEVA (Golay 1972), Strömgren (Strömgren 1956), Tycho-2 (Høg et al. 2000), 2MASS (Cutri et al. 2003) and SDSS (Fukugita et al. 1996) systems, (far-IR) from the InfraRed Astronomy Satellite (IRAS, Neugebauer et al. 1984), the AKARI satellite (Murakami et al. 2007), the DIRBE instrument on the COBE satellite (Smith et al. 2004), the WISE satellite (Cutri \& et al. 2012) and the Midcourse Space Experiment (MSX, Price 1995). In addition, we included some newly acquired near-IR photometry from the South African Astronomical Observatory (SAAO).
For sources with small variability amplitudes, the photometry retrieved in this way is generally sufficient for our purposes. In a few cases, however, some additional photometry was added as well (see below). For the sources with large-amplitude flux variations, more care was taken to only select photometry at specific epochs. To this end, we also made an extensive search through the literature to find multicolour photometry for which a phase attribution could be made with the light curves from the ASAS and AAVSO databases.

Finally, we note that for some stars, measurements in specific bands of certain systems were not retained, e.g. due to saturation issues. This is particularly relevant for WISE data (mainly $W 1$ and $W 2$ ), for which we employed the following limits: $W_{1}>2.5$, $W_{2}>2.0, W_{3}>-2.5$ and $W_{4}>-3.5$. Upper limits were not retained either (e.g. for some IRAS.F100 measurements).

Table A.1. Full reference list to the photometric data used to construct the SEDs.

\begin{tabular}{|c|c|c|c|c|}
\hline Star & Photometric system & Retained bands & $\begin{array}{l}\text { Vizier catalogue } \\
\text { or bibl. reference }\end{array}$ & Note \\
\hline \multicolumn{5}{|c|}{ Stars with negligible variability } \\
\hline \multirow[t]{13}{*}{ IRAS $07008+1050$} & 2MASS & $J, H, K \mathrm{~s}$ & II/246/out & \\
\hline & IRAS & $F 12, F 25, F 60$ & $\mathrm{II} / 275 / \mathrm{fsr}$ & \\
\hline & TD1 & $1965,2365,2740$ & II/59B/catalogue & \\
\hline & TYCHO-2 & $B T, V T$ & $\mathrm{I} / 259 /$ tyc2 & \\
\hline & JOHNSON & $B, V$ & V/137C/XHIP & \\
\hline & COUSINS & $R, I$ & V/137C/XHIP & \\
\hline & JOHNSON & $B, V$ & $\mathrm{I} / 280 \mathrm{~B} / \mathrm{ascc}$ & \\
\hline & JOHNSON & $V$ & II/271A/patch2 & \\
\hline & COUSINS & $I$ & $\mathrm{II} / 271 \mathrm{~A} / \mathrm{patch} 2$ & \\
\hline & AKARI & WIDES & II/298/fis & \\
\hline & AKARI & $S 9 W, L 18 W$ & II/297/irc & \\
\hline & WISE & $W 1, W 2, W 3, W 4$ & II/311/wise & \\
\hline & GENEVA & $U, B, V, B 1, B 2, V 1, G$ & $\mathrm{GCPD}^{a}$ & \\
\hline \multirow{10}{*}{ IRAS 08544-4431 } & 2MASS & $J, H, K \mathrm{~s}$ & II/246/out & \\
\hline & IRAS & $F 12, F 25, F 60$ & $\mathrm{II} / 275 / \mathrm{fsr}$ & \\
\hline & TYCHO-2 & $B T, V T$ & $\mathrm{I} / 259 /$ tyc 2 & \\
\hline & JOHNSON & $U, B, V$ & de Ruyter et al. (2006) & \\
\hline & COUSINS & $R, I$ & de Ruyter et al. (2006) & \\
\hline & AKARI & N60, WIDES, WIDEL & II/298/fis & \\
\hline & AKARI & $S 9 W, L 18 W$ & II/297/irc & \\
\hline & MSX & $B 1, B 2, A, C, D, E$ & V/114/msx6_gp & \\
\hline & WISE & $W 3, W 4$ & II/311/wise & \\
\hline & SAAO & $J, H, K, L$ & de Ruyter et al. (2006) & \\
\hline \multirow[t]{11}{*}{ IRAS 09256-6324 } & 2MASS & $J, H, K \mathrm{~s}$ & II/246/out & \\
\hline & IRAS & $F 12, F 25, F 60, F 100$ & $\mathrm{II} / 275 / \mathrm{fsr}$ & \\
\hline & TYCHO-2 & $B T, V T$ & $\mathrm{I} / 259 /$ tyc 2 & \\
\hline & JOHNSON & $J, H, K, L, M$ & II/237/colours & \\
\hline & AKARI & N60, WIDES, WIDEL & II/298/fis & \\
\hline & AKARI & $S 9 W, L 18 W$ & II/297/irc & \\
\hline & STROMGREN & $Y, B, V, U$ & II/215/catalogue & \\
\hline & DIRBE & F4_9, F12, F25 & $\mathrm{J} / \mathrm{ApJS} / 154 / 673 / \mathrm{DIRBE}$ & \\
\hline & WISE & $W \overline{1}, W 3, W 4$ & II/311/wise & \\
\hline & JOHNSON & $B, V$ & Pollard et al. (1996) & max. RVb phase \\
\hline & COUSINS & $R, I$ & Pollard et al. (1996) & max. RVb phase \\
\hline
\end{tabular}

Notes. ${ }^{(a)}$ Mermilliod et al. (1997); ${ }^{(b)}$ data taken from the archive of the Mercator telescope. We did not use the values from de Ruyter et al. (2006) in this case because they correspond to a minimum RVb phase; ${ }^{(c)}$ for this star the mean $J H K L$ values were used in both pulsation phases. 
Table A.1. continued.

\begin{tabular}{|c|c|c|c|c|}
\hline Star & Photometric system & Retained bands & $\begin{array}{l}\text { Vizier catalogue } \\
\text { or bibl. reference }\end{array}$ & Note \\
\hline \multirow[t]{14}{*}{ IRAS 10158-2844 } & 2MASS & $J, H, K \mathrm{~s}$ & II/246/out & \\
\hline & IRAS & $F 12, F 25, F 60$ & II/125/main & \\
\hline & TYCHO2 & $B T, V T$ & $\mathrm{I} / 259 /$ tyc2 & \\
\hline & JOHNSON & $B, V$ & V/137C/XHIP & \\
\hline & COUSINS & $R, I$ & V/137C/XHIP & \\
\hline & JOHNSON & $B, V$ & $\mathrm{I} / 280 \mathrm{~B} / \mathrm{ascc}$ & \\
\hline & JOHNSON & $B, V$ & II/168/ubvmeans & \\
\hline & AKARI & WIDES & II/298/fis & \\
\hline & AKARI & $S 9 W, L 18 W$ & II/297/irc & \\
\hline & STROMGREN & $Y, B, V, U$ & $\mathrm{~J} / \mathrm{A}+\mathrm{A} / 373 / 625 /$ table 2 & \\
\hline & DIRBE & $F 1 \_25, F 2 \_2, F 3 \_5, F 4 \_9$ & J/ApJS/154/673/DIRBE & \\
\hline & WISE & $W 3, W 4$ & II/311/wise & \\
\hline & GENEVA & $U, B, V, B 1, B 2, V 1, G$ & $\mathrm{GCPD}^{a}$ & \\
\hline & STROMGREN & $Y, B, V, U$ & $\mathrm{GCPD}^{a}$ & \\
\hline \multirow{8}{*}{ IRAS 10174-5704 } & 2MASS & $J, H, K \mathrm{~s}$ & II/246/out & \\
\hline & IRAS & $F 12, F 25$ & $\mathrm{II} / 275 / \mathrm{fsr}$ & \\
\hline & TYCHO2 & $B T, V T$ & $\mathrm{I} / 259 /$ tyc 2 & \\
\hline & AKARI & N60, WIDES & II/298/fis & \\
\hline & AKARI & $S 9 W, L 18 W$ & II/297/irc & \\
\hline & MSX & $B 1, B 2, A, C, D, E$ & V/114/msx6_gp & \\
\hline & WISE & $W 1, W 2, W 3, W 4$ & II/311/wise & \\
\hline & SAAO & $J, H, K, L$ & - & P. Whitelock \\
\hline \multirow[t]{13}{*}{ IRAS 10456-5712 } & 2MASS & $J, H, K \mathrm{~s}$ & II/246/out & \\
\hline & IRAS & $F 12, F 25, F 60, F 100$ & II/125/main & \\
\hline & TYCHO-2 & $B T, V T$ & $\mathrm{I} / 259 /$ tyc2 & \\
\hline & JOHNSON & $B, V$ & V/137C/XHIP & \\
\hline & COUSINS & $I$ & V/137C/XHIP & \\
\hline & JOHNSON & $B, V$ & $\mathrm{I} / 280 \mathrm{~B} / \mathrm{ascc}$ & \\
\hline & JOHNSON & $U, B, V$ & II/168/ubvmeans & \\
\hline & JOHNSON & $B, V$ & II/5A/data & \\
\hline & AKARI & N60, WIDES & II/298/fis & \\
\hline & AKARI & $S 9 W, L 18 W$ & II/297/irc & \\
\hline & MSX & $B 1, B 2, A, C, D, E$ & V/114/msx6_gp & \\
\hline & WISE & $W 3, W 4$ & II/311/wise & \\
\hline & SAAO & $J, H, K, L$ & - & P. Whitelock \\
\hline \multirow{10}{*}{ IRAS 11385-5517 } & 2MASS & $J, H, K \mathrm{~s}$ & II/246/out & \\
\hline & IRAS & $F 12, F 25, F 60, F 100$ & $\mathrm{II} / 275 / \mathrm{fsr}$ & \\
\hline & TYCHO-2 & $B T, V T$ & $\mathrm{I} / 259 /$ tyc 2 & \\
\hline & JOHNSON & $U, B, V$ & II/168/ubvmeans & \\
\hline & JOHNSON & $U, B, V, J, H, K, L, M$ & II/237/colours & \\
\hline & COUSINS & $R, I$ & V/137/XHIP & \\
\hline & AKARI & N60, WIDES, WIDEL, N160 & II/298/fis & \\
\hline & AKARI & $S 9 W, L 18 W$ & II/297/irc & \\
\hline & STROMGREN & $Y, B, V, U$ & II/215/catalogue & \\
\hline & ALMA & Band6 & Olofsson et al. (2015) & \\
\hline \multirow{11}{*}{ IRAS 12222-4652 } & 2MASS & $J, H, K \mathrm{~s}$ & II/246/out & \\
\hline & IRAS & $F 12, F 25, F 60, F 100$ & II/125/main & \\
\hline & TYCHO-2 & $B T, V T$ & $\mathrm{I} / 259 /$ tyc2 & \\
\hline & JOHNSON & $B, V$ & $\mathrm{I} / 280 \mathrm{~B} / \mathrm{ascc}$ & \\
\hline & AKARI & N60, WIDES & II/298/fis & \\
\hline & AKARI & $S 9 W, L 18 W$ & II/297/irc & \\
\hline & STROMGREN & $Y, B, V, U$ & II/215/catalogue & \\
\hline & WISE & $W 1, W 2, W 3, W 4$ & II/311/wise & \\
\hline & GENEVA & $U, B, V, B 1, B 2, V 1, G$ & $\mathrm{GCPD}^{a}$ & \\
\hline & STROMGREN & $Y, B, V, U$ & $\mathrm{GCPD}^{a}$ & \\
\hline & SAAO & $J, H, K, L$ & - & P. Whitelock \\
\hline \multirow[t]{9}{*}{ IRAS 15469-5311 } & 2MASS & $J, H, K \mathrm{~s}$ & II/246/out & \\
\hline & IRAS & $F 12, F 25, F 60$ & II/125/main & \\
\hline & TYCHO-2 & $B T, V T$ & $\mathrm{I} / 259 /$ tyc2 & \\
\hline & JOHNSON & $B, V$ & $\mathrm{I} / 280 \mathrm{~B} / \mathrm{ascc}$ & \\
\hline & AKARI & N60, WIDES & II/298/fis & \\
\hline & AKARI & $S 9 W, L 18 W$ & II/297/irc & \\
\hline & MSX & $B 1, B 2, A, C, D, E$ & V/114/msx6_gp & \\
\hline & WISE & $W 1, W 3, W 4$ & II/311/wise & \\
\hline & SAAO & $J, H, K, L$ & - & P. Whitelock \\
\hline
\end{tabular}


Table A.1. continued.

\begin{tabular}{|c|c|c|c|c|}
\hline Star & Photometric system & Retained bands & $\begin{array}{l}\text { Vizier catalogue } \\
\text { or reference }\end{array}$ & Note \\
\hline \multirow{16}{*}{ IRAS $17535+2603$} & 2MASS & $J, H, K \mathrm{~s}$ & II/246/out & \\
\hline & IRAS & $F 12, F 25, F 60, F 100$ & II/125/main & \\
\hline & TD1 & $1965,2365,2740$ & II/59B/catalogue & \\
\hline & ANS & $18,25,33$ & II/97/ans & \\
\hline & TYCHO2 & $B T, V T$ & $\mathrm{I} / 259 /$ tyc 2 & \\
\hline & JOHNSON & $B, V$ & $\mathrm{I} / 280 \mathrm{~B} / \mathrm{ascc}$ & \\
\hline & JOHNSON & $U, B, V, R, I$ & II/7A/catalogue & \\
\hline & JOHNSON & $B, V, R, I, H, K, L, M, N$ & II/237/colours & \\
\hline & JOHNSON & $U, B, V$ & II/168/ubvmeans & \\
\hline & AKARI & N60, WIDES, WIDEL, N160 & II/298/fis & \\
\hline & AKARI & $S 9 W, L 18 W$ & II/297/irc & \\
\hline & DIRBE & $F 3 \_5, F 4 \_9, F 12$ & J/ApJS/154/673/DIRBE & \\
\hline & WISE & $W 4$ & II/311/wise & \\
\hline & GENEVA & $U, B, V, B 1, B 2, V 1, G$ & $\mathrm{GCPD}^{a}$ & \\
\hline & STROMGREN & $Y, B, V, U$ & $\mathrm{GCPD}^{a}$ & \\
\hline & VILNIUS & $V, Z, S, Y, X, P, U$ & $\mathrm{GCPD}^{a}$ & \\
\hline \multirow{11}{*}{ IRAS $19125+0343$} & 2MASS & $J, H, K \mathrm{~s}$ & II/246/out & \\
\hline & IRAS & $F 12, F 25, F 60$ & II/125/main & \\
\hline & TYCHO-2 & $B T, V T$ & $\mathrm{I} / 259 / \mathrm{tyc} 2$ & \\
\hline & JOHNSON & $B, V$ & $\mathrm{I} / 280 \mathrm{~B} / \mathrm{ascc}$ & \\
\hline & JOHNSON & $V$ & $\mathrm{II} / 271 \mathrm{~A} /$ patch 2 & \\
\hline & COUSINS & $I$ & $\mathrm{II} / 271 \mathrm{~A} /$ patch 2 & \\
\hline & SDSS & $\mathrm{RP}$ & I/304/out & \\
\hline & AKARI & $S 9 W, L 18 W$ & II/297/irc & \\
\hline & MSX & $B 2, A, C, D, E$ & V/114/msx6_gp & \\
\hline & WISE & $W 1, W 3, W 4$ & II/311/wise & \\
\hline & GENEVA & $U, B, V, B 1, B 2, V 1, G$ & de Ruyter et al. (2006) & \\
\hline \multirow{12}{*}{ IRAS 22327-1731 } & 2MASS & $J, H, K \mathrm{~s}$ & II/246/out & \\
\hline & IRAS & $F 12, F 25, F 60$ & II/125/main & \\
\hline & TYCHO-2 & $B T, V T$ & $\mathrm{I} / 259 /$ tyc2 & \\
\hline & JOHNSON & $B, V$ & V/137C/XHIP & \\
\hline & COUSINS & $R, I$ & V/137C/XHIP & \\
\hline & JOHNSON & $B, V$ & $\mathrm{I} / 280 \mathrm{~B} / \mathrm{ascc}$ & \\
\hline & JOHNSON & $U, B, V, J, H, K, L$ & $\mathrm{II} / 237 /$ colours & \\
\hline & AKARI & WIDES & II/298/fis & \\
\hline & AKARI & $S 9 W, L 18 W$ & II/297/irc & \\
\hline & WISE & $W 1, W 2, W 3, W 4$ & II/311/wise & \\
\hline & STROMGREN & $Y, B, V, U$ & $\mathrm{GCPD}^{a}$ & \\
\hline & GENEVA & $U, B, V, B 1, B 2, V 1, G$ & de Ruyter et al. (2006) & max. RVb phase \\
\hline \multicolumn{5}{|c|}{ Stars with significant variability } \\
\hline \multirow[t]{9}{*}{ IRAS $04440+2605$} & JOHNSON & $B, V$ & Wahlgren (1992) & max. puls. and RVb phase \\
\hline & JOHNSON & $B, V$ & Wahlgren (1992) & min. puls. and max. RVb phase \\
\hline & JOHNSON & $J, H, K, L, M$ & Taranova et al. (2009) & max. puls. and RVb phase \\
\hline & GENEVA & $U, B, V, B 1, B 2, V 1, G$ & de Ruyter et al. (2006) & max. puls. and RVb phase \\
\hline & GENEVA & $U, B, V, B 1, B 2, V 1, G$ & $-b$ & min. puls. and max RVb. phase \\
\hline & AKARI & N60, WIDES & II/298/fis & \\
\hline & AKARI & $S 9 W, L 18 W$ & II/297/irc & \\
\hline & WISE & $W 1, W 2, W 3, W 4$ & II/311/wise & \\
\hline & 2MASS & $J, H, K$ & II/246/out & \\
\hline \multirow[t]{17}{*}{ IRAS 07284-0940 } & JOHNSON & $B, V$ & Pollard et al. (1996) & max. puls. and RVb phase \\
\hline & COUSINS & $R, I$ & Pollard et al. (1996) & max. puls. and RVb phase \\
\hline & GENEVA & $U, B, V, B 1, B 2, V 1, G$ & de Ruyter et al. (2006) & max. puls. and RVb phase \\
\hline & JOHNSON & $U, B, V$ & Preston et al. (1963) & max. puls. and RVb phase \\
\hline & JOHNSON & $U, B, V$ & Preston et al. (1963) & max. puls. and RVb phase \\
\hline & SAAO & $J, H, K, L$ & Evans (1985) & max. puls. and RVb phase \\
\hline & JOHNSON & $B, V$ & Pollard et al. (1996) & min. puls. and max. RVb phase \\
\hline & COUSINS & $R, I$ & Pollard et al. (1996) & min. puls. and max. RVb phase \\
\hline & GENEVA & $U, B, V, B 1, B 2, V 1, G$ & de Ruyter et al. (2006) & min. puls. and max. RVb phase \\
\hline & JOHNSON & $U, B, V$ & Preston et al. (1963) & min. puls. and max. RVb phase \\
\hline & SAAO & $J, H, K, L$ & Evans (1985) & min. puls. and max. RVb phase \\
\hline & 2MASS & $J, H, K$ & II/246/out & \\
\hline & IRAS & $F 12, F 25, F 60, F 100$ & $\mathrm{II} / 125 /$ main & \\
\hline & AKARI & N60, WIDES & II/298/fis & \\
\hline & AKARI & $S 9 W, L 18 W$ & II/297/irc & \\
\hline & MSX & $B 2, A, C, D, E$ & V/114/msx6_gp & \\
\hline & WISE & $W 1, W 3, W 4$ & II/311/wise & \\
\hline
\end{tabular}


Table A.1. continued.

\begin{tabular}{|c|c|c|c|c|}
\hline Star & Photometric system & Retained bands & $\begin{array}{l}\text { Vizier catalogue } \\
\text { or reference }\end{array}$ & Note \\
\hline \multirow[t]{11}{*}{ IRAS 08011-3627 } & JOHNSON & $B, V$ & Pollard et al. (1996) & max. puls. and RVb phase \\
\hline & GENEVA & $U, B, V, B 1, B 2, V 1, G$ & de Ruyter et al. (2006) & max. puls. and RVb phase \\
\hline & SAAO & $J, H, K, L$ & Evans (1985) & max. puls. and RVb phase \\
\hline & JOHNSON & $B, V$ & Pollard et al. (1996) & min. puls. and max. RVb phase \\
\hline & SAAO & $J, H, K, L$ & Evans (1985) & min. puls. and max. RVb phase \\
\hline & 2MASS & $J, H, K \mathrm{~s}$ & II/246/out & \\
\hline & IRAS & $F 12, F 25, F 60$ & II/125/main & \\
\hline & AKARI & WIDES & $\mathrm{II} / 298 / \mathrm{fis}$ & \\
\hline & AKARI & $S 9 W, L 18 W$ & II/297/irc & \\
\hline & DIRBE & $F 1 \_25, F 2 \_2, F 3 \_5, F 4 \_9$ & J/ApJS/154/673/DIRBE & \\
\hline & WISE & $W 3, W 4$ & $\mathrm{II} / 311 /$ wise & \\
\hline \multirow[t]{11}{*}{ IRAS 12185-4856 } & JOHNSON & $U, B, V$ & Goldsmith et al. (1987) & max. puls. and RVb phase \\
\hline & COUSINS & $R, I$ & Goldsmith et al. (1987) & max. puls. and RVb phase \\
\hline & SAAO & $J, H, K, L$ & Goldsmith et al. (1987) & max. puls. and RVb phase \\
\hline & JOHNSON & $U, B, V$ & Goldsmith et al. (1987) & min. puls. and max. RVb phase \\
\hline & COUSINS & $R, I$ & Goldsmith et al. (1987) & min. puls. and max. RVb phase \\
\hline & SAAO & $J, H, K, L$ & Goldsmith et al. (1987) & min. puls. and max. RVb phase \\
\hline & 2MASS & $J, H, K \mathrm{~s}$ & II/246/out & \\
\hline & IRAS & $F 12, F 25, F 60$ & II/125/main & \\
\hline & AKARI & WIDES & II/298/fis & \\
\hline & AKARI & $S 9 W, L 18 W$ & II/297/irc & \\
\hline & WISE & $W 1, W 2, W 3, W 4$ & II/311/wise & \\
\hline \multirow[t]{10}{*}{ IRAS $17038-4815$} & JOHNSON & $U, B, V$ & de Ruyter et al. (2006) & max. puls. and RVb phase \\
\hline & COUSINS & $R, I$ & de Ruyter et al. (2006) & max. puls. and RVb phase \\
\hline & JOHNSON & $U, B, V$ & de Ruyter et al. (2006) & min. puls. and max. RVb phase \\
\hline & COUSINS & $R, I$ & de Ruyter et al. (2006) & min. puls. and max. RVb phase \\
\hline & 2MASS & $J, H, K \mathrm{~s}$ & II/246/out & \\
\hline & IRAS & $F 12, F 25, F 60$ & II/125/main & \\
\hline & AKARI & WIDES, WIDEL & II/298/fis & \\
\hline & AKARI & $S 9 W, L 18 W$ & II/297/irc & \\
\hline & MSX & $A, C, D, E$ & V/114/msx6_gp & \\
\hline & WISE & $W 1, W 2, W 3, W 4$ & II/311/wise & \\
\hline \multirow{11}{*}{ IRAS $17243-4348$} & JOHNSON & $U, B, V$ & de Ruyter et al. (2006) & max. puls. phase \\
\hline & COUSINS & $R, I$ & de Ruyter et al. (2006) & max. puls. phase \\
\hline & SAAO & $J, H, K, L$ & de Ruyter et al. (2006) & T. Lloyd Evans (mean) ${ }^{c}$ \\
\hline & JOHNSON & $U, B, V$ & de Ruyter et al. (2006) & min. puls. phase \\
\hline & COUSINS & $R, I$ & de Ruyter et al. (2006) & min. puls. phase \\
\hline & 2MASS & $J, H, K \mathrm{~s}$ & II/246/out & \\
\hline & IRAS & $F 12, F 25, F 60$ & $\mathrm{II} / 275 / \mathrm{fsr}$ & \\
\hline & AKARI & WIDES & II/298/fis & \\
\hline & AKARI & $S 9 W, L 18 W$ & II/297/irc & \\
\hline & MSX & $A, C, D, E$ & V/114/msx6_gp & \\
\hline & WISE & $W 1, W 2, W 3, W 4$ & II/311/wise & \\
\hline \multirow[t]{11}{*}{ IRAS $18281+2149$} & GENEVA & $U, B, V, B 1, B 2, V 1, G$ & de Ruyter et al. (2006) & max. puls. phase \\
\hline & JOHNSON & $U, B, V$ & Zsoldos (1993) & max. puls. phase \\
\hline & JOHNSON & $J, H, K, L$ & Taranova et al. (2010) & max. puls. phase \\
\hline & GENEVA & $U, B, V, B 1, B 2, V 1, G$ & de Ruyter et al. (2006) & min. puls. phase \\
\hline & JOHNSON & $U, B, V$ & Zsoldos (1993) & min. puls. phase \\
\hline & JOHNSON & $J, H, K, L$ & Taranova et al. (2010) & min. puls. phase \\
\hline & 2MASS & $J, H, K s$ & II/246/out & \\
\hline & IRAS & $F 12, F 25, F 60, F 100$ & II/125/main & \\
\hline & AKARI & N60, WIDES, WIDEL & $\mathrm{II} / 298 / \mathrm{fis}$ & \\
\hline & AKARI & $S 9 W, L 18 W$ & II/297/irc & \\
\hline & WISE & $W 1, W 2, W 3, W 4$ & II/311/wise & \\
\hline
\end{tabular}




\section{Appendix B: Additional figures}
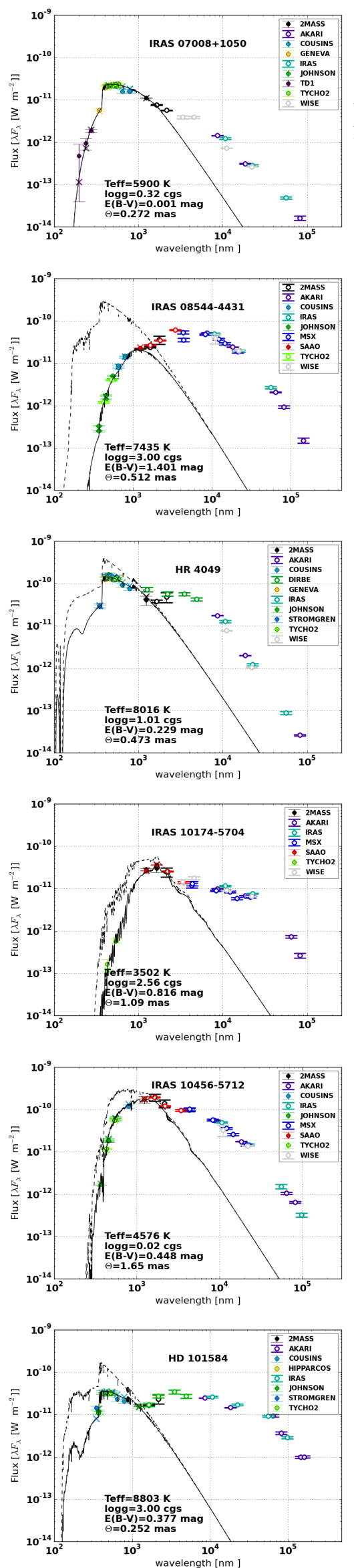
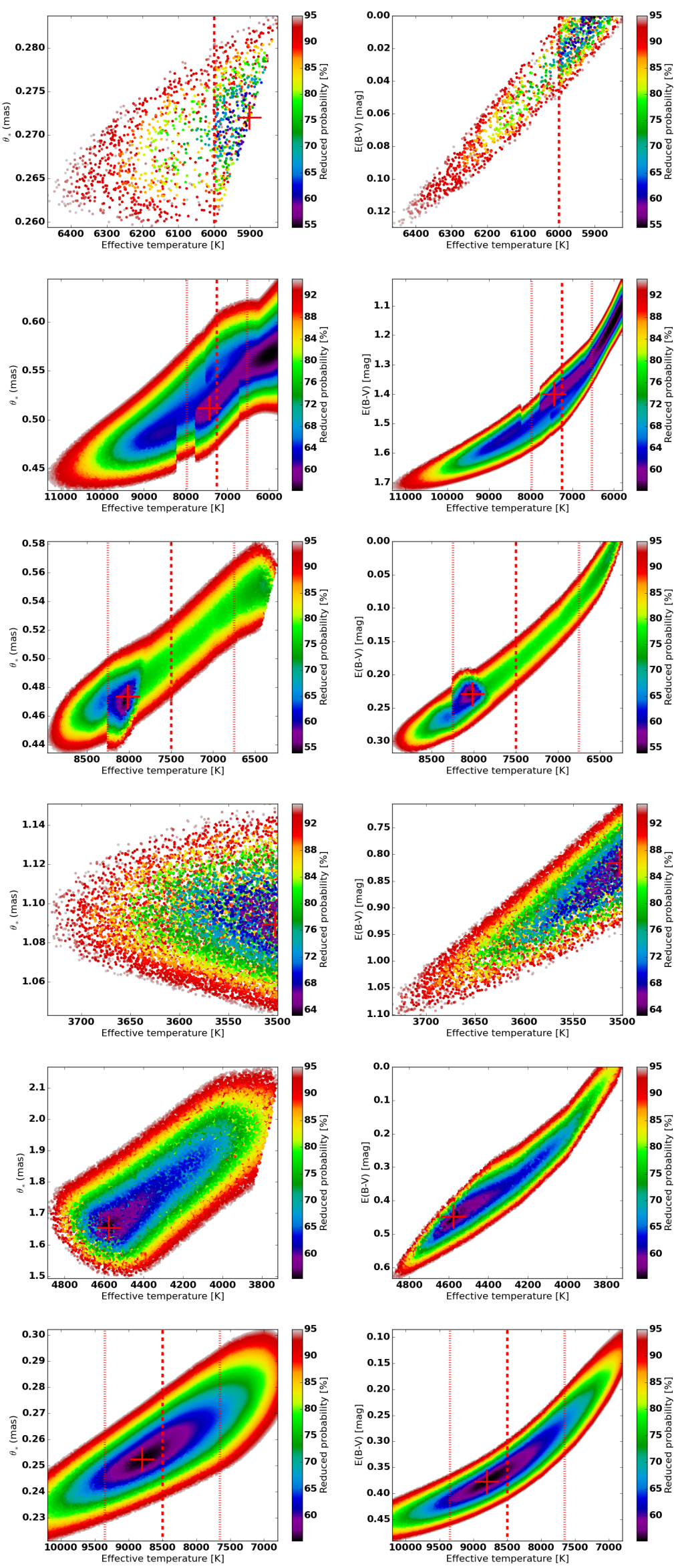

Fig. B.1. Same as Fig. 2 of the main text, for the other non-variable sources in our sample. 
M. Hillen et al.: MIDI on post-AGB binaries
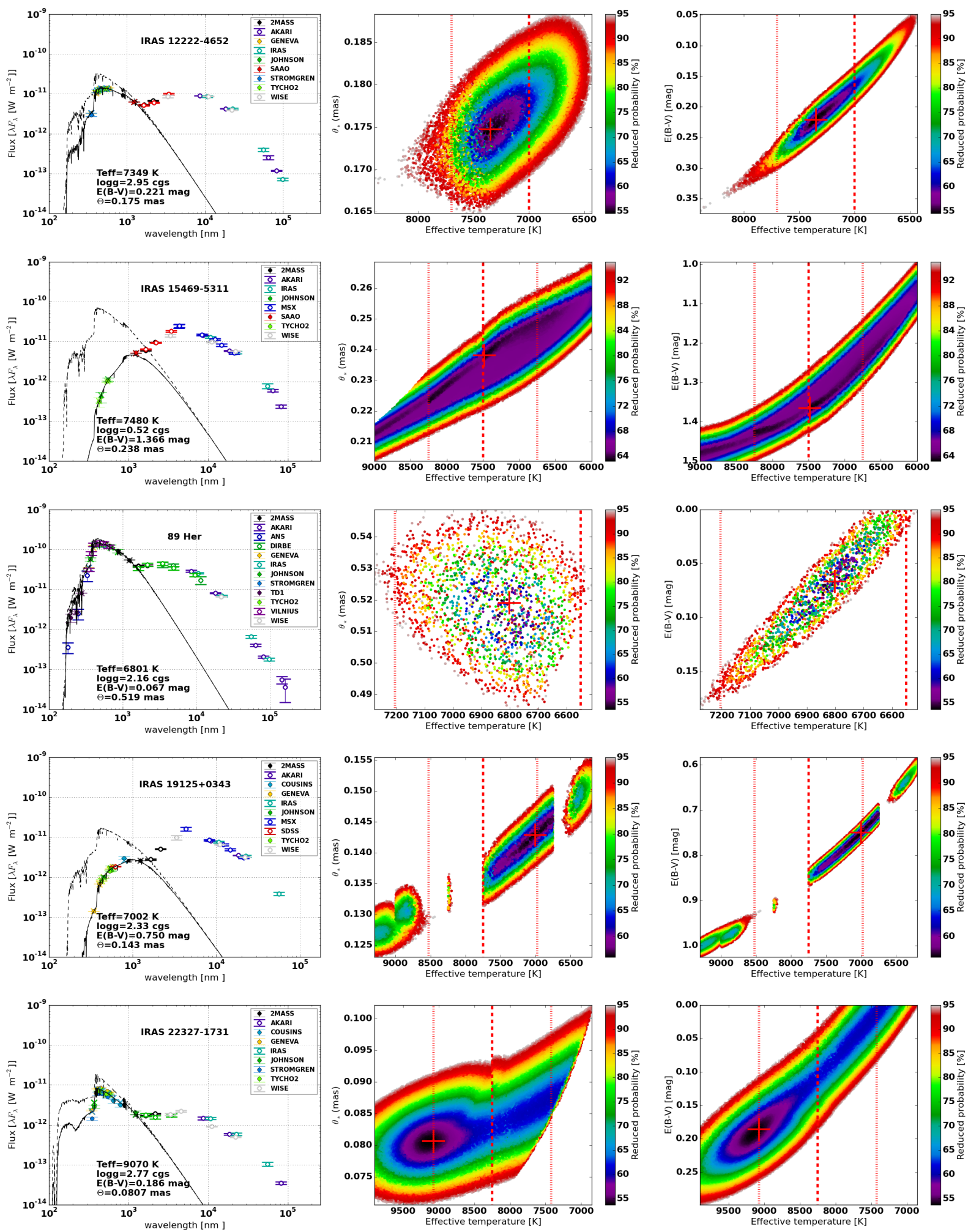

Fig. B.1. continued. 

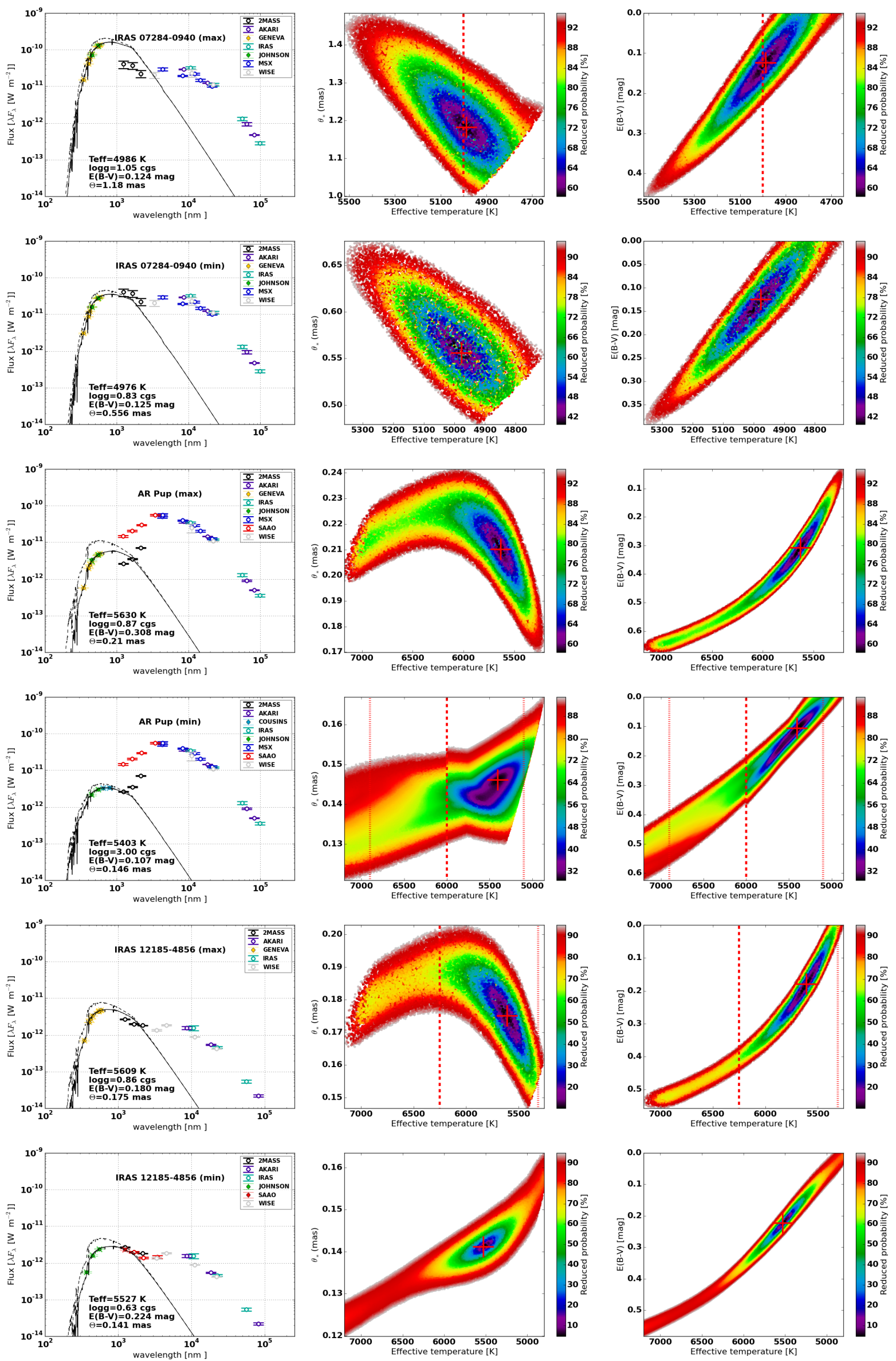

Fig. B.2. Same as Fig. 3 of the main text, for the variable sources in our sample. 
M. Hillen et al.: MIDI on post-AGB binaries
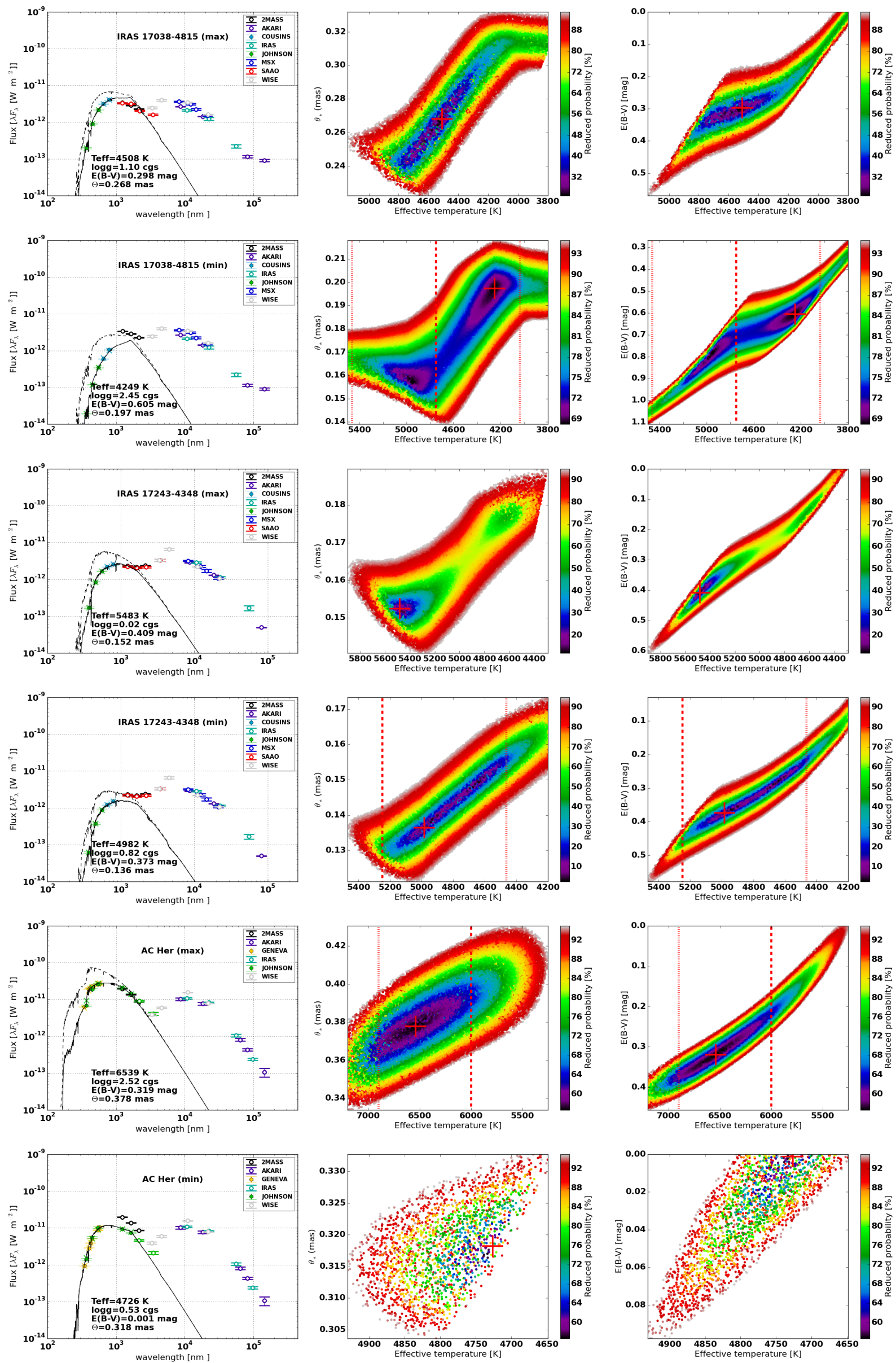

Fig. B.2. continued. 

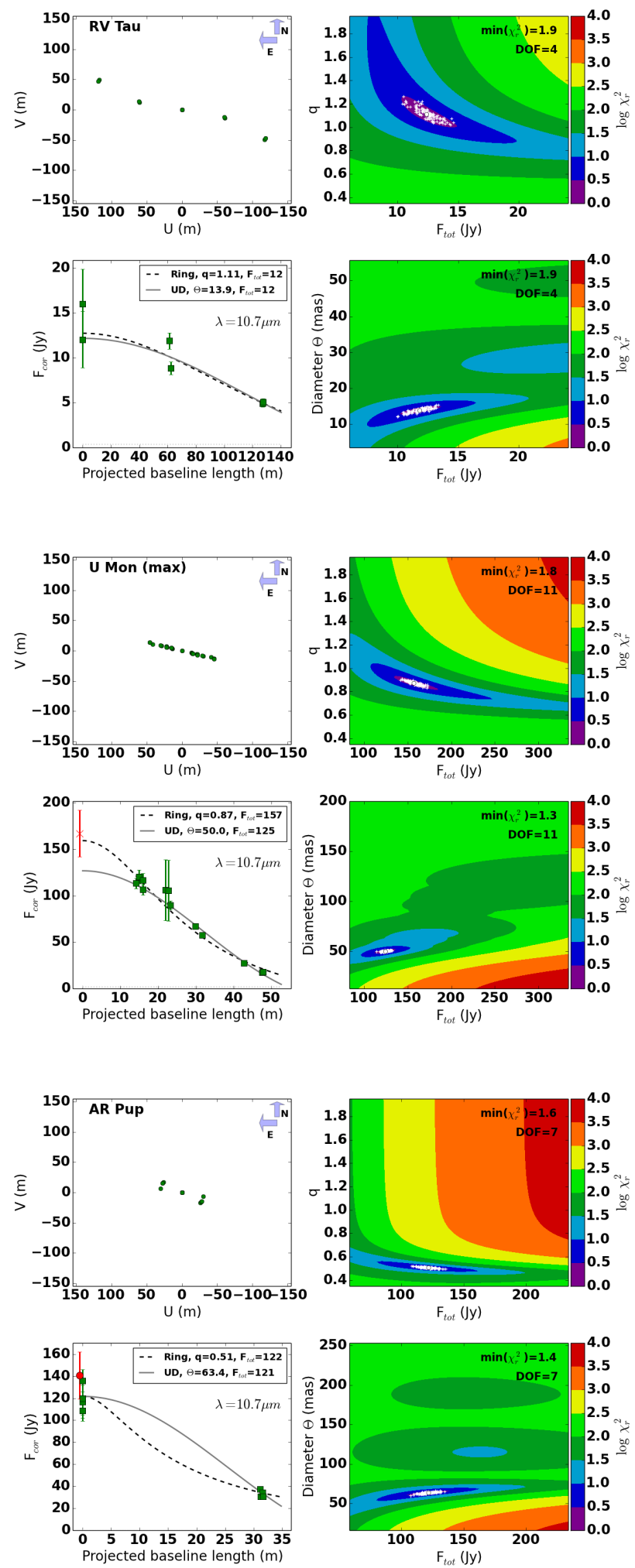
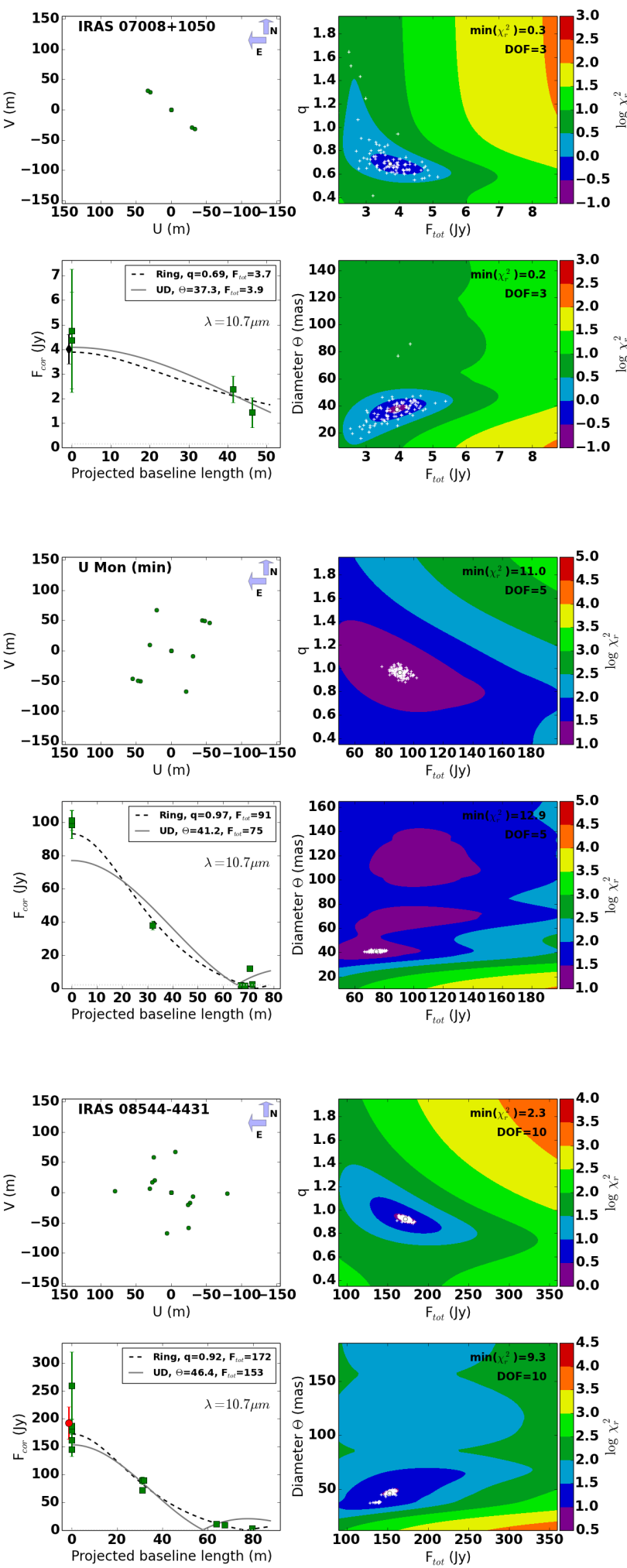

Fig. B.3. Same as Fig 6 of the main text, but for the other sources in the sample. 

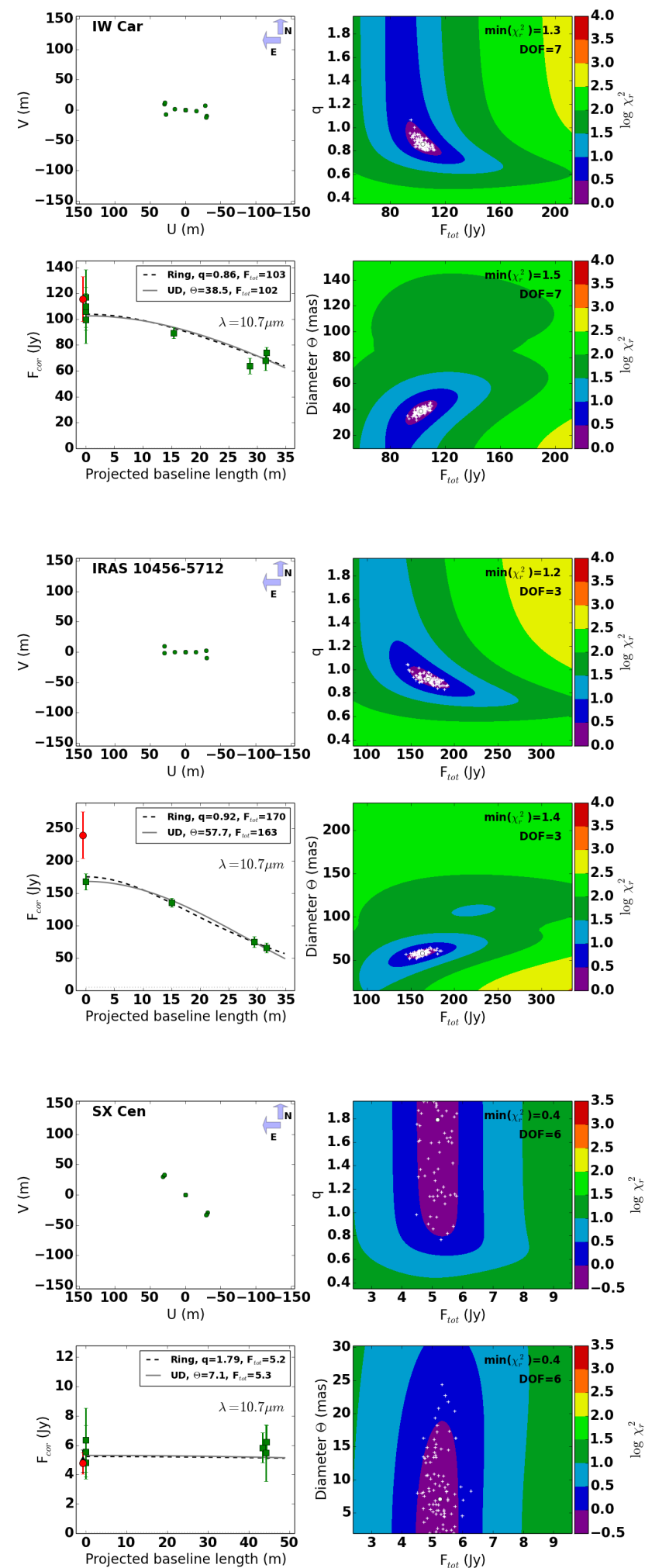
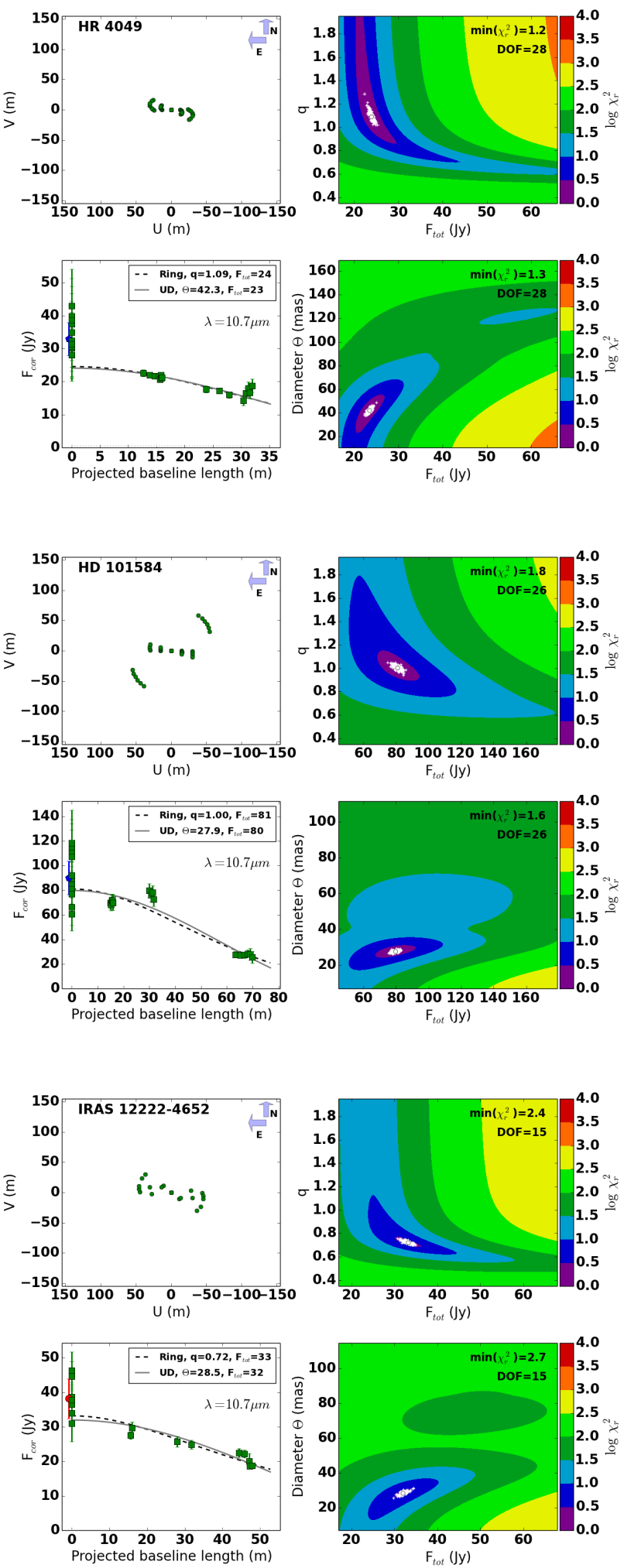

Fig. B.3. continued. 

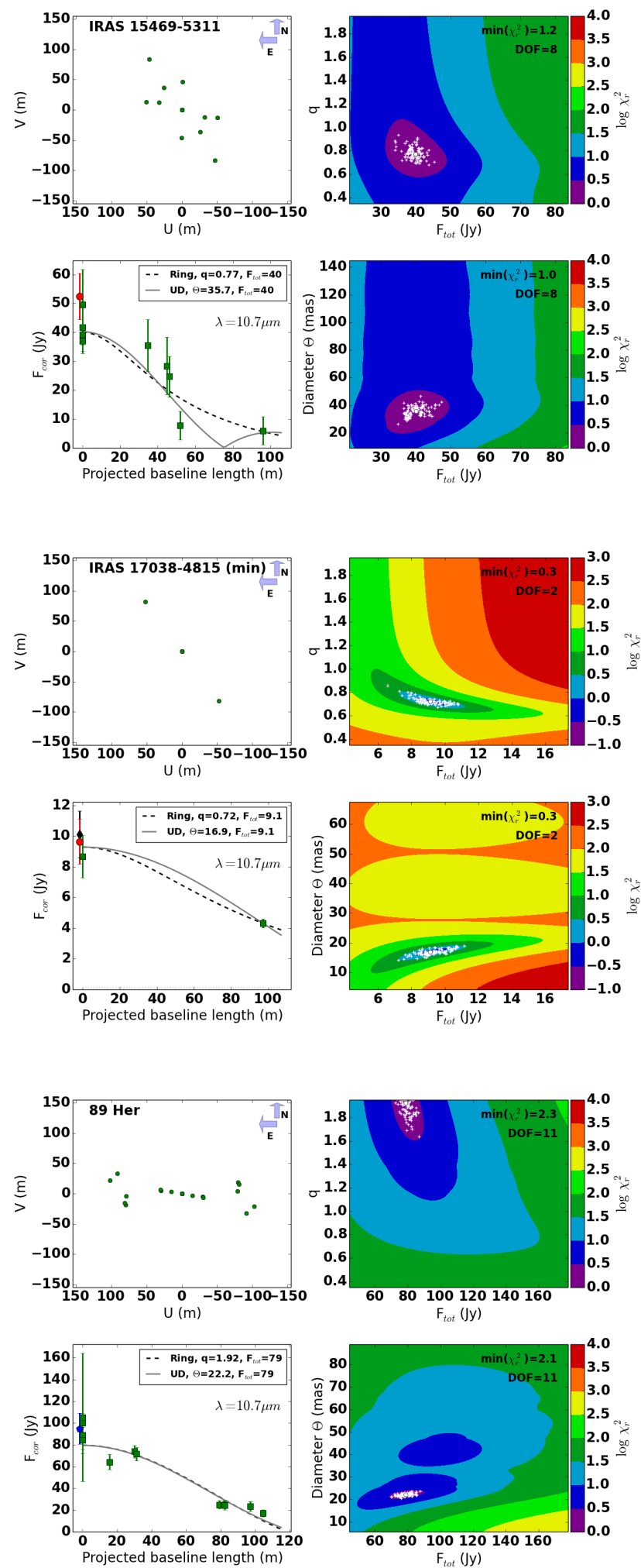
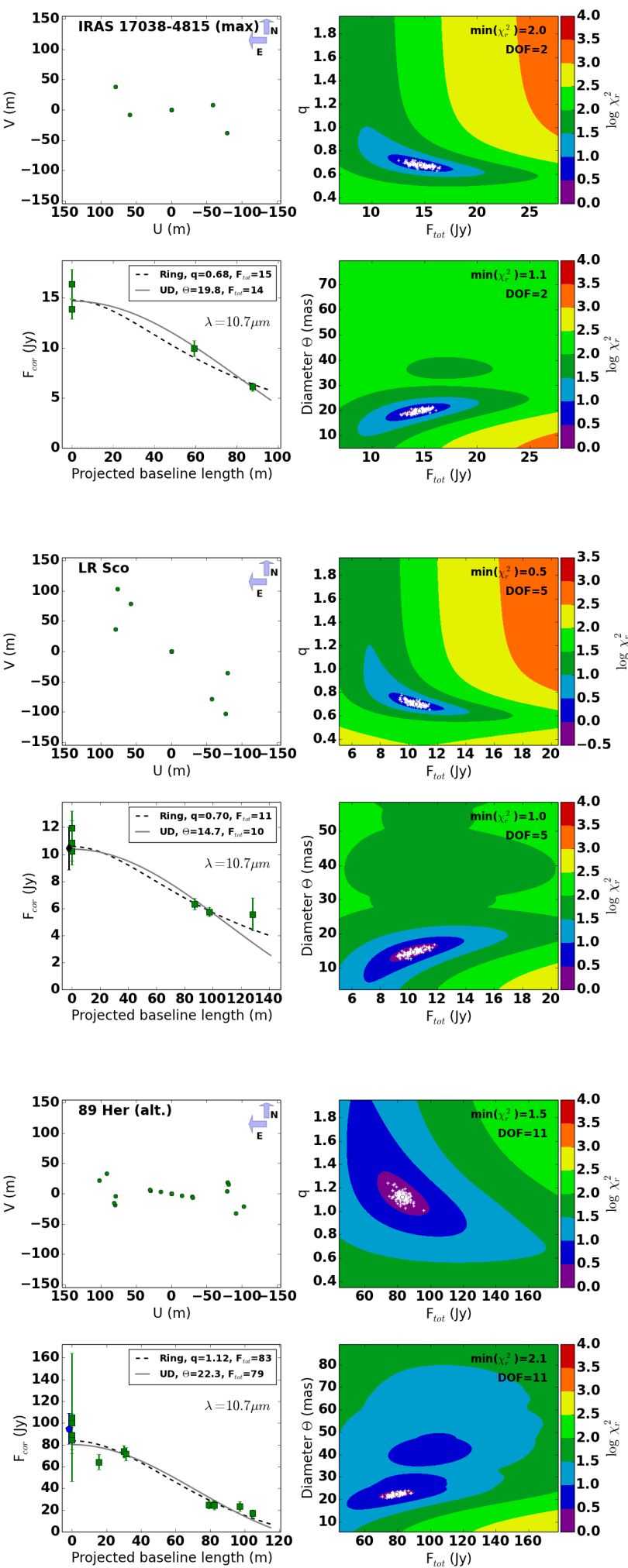

Fig. B.4. Lower right panel: result for the $89 \mathrm{Her}$ data set with alternative stellar parameters that take the large detected fraction of scattered light into account (Hillen et al. 2013). 
M. Hillen et al.: MIDI on post-AGB binaries
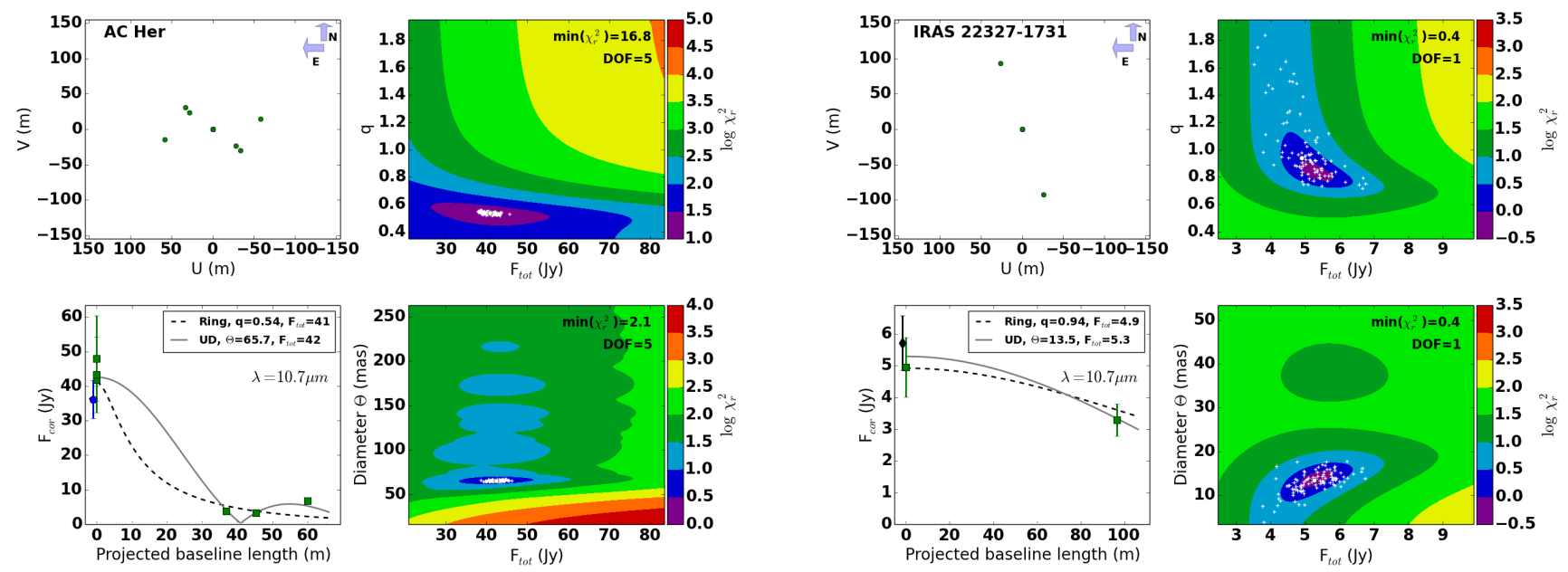

Fig. B.4. continued. 